\title{
X-shooter observations of low-mass stars in the $\eta$ Chamaeleontis association ${ }^{\star}$
}

\author{
Michael Rugel $^{1}$, Davide Fedele ${ }^{2}$, and Gregory Herczeg ${ }^{3}$ \\ 1 Max Planck Institute for Astronomy, Königstuhl 17, 69117 Heidelberg, Germany \\ e-mail: rugel@mpia.de \\ 2 INAF-Osservatorio Astrofisico di Arcetri, L.go E. Fermi 5, 50125 Firenze, Italy \\ 3 Kavli Institute for Astronomy and Astrophysics, Peking University, Yi He Yuan Lu 5, Haidian Qu, 100871 Beijing, PR China
}

Received 22 November 2016 / Accepted 7 September 2017

\begin{abstract}
The nearby $\eta$ Chamaeleontis association is a collection of 4-10 Myr old stars with a disk fraction of 35-45\%. In this study, the broad wavelength coverage of VLT/X-shooter is used to measure the stellar and mass accretion properties of 15 low-mass stars in the $\eta$ Chamaeleontis association. For each star, the observed spectrum is fitted with a non-accreting stellar template and an accretion spectrum obtained from assuming a plane-parallel hydrogen slab. Five of the eight stars with an IR disk excess show excess UV emission, indicating ongoing accretion. The accretion rates measured here are similar to those obtained from previous measurements of excess UV emission, but tend to be higher than past measurements from $\mathrm{H} \alpha$ modeling. The mass accretion rates are consistent with those of other young star forming regions.
\end{abstract}

Key words. stars: pre-main sequence - stars: low-mass - stars: variables: T Tauri, Herbig $\mathrm{Ae} / \mathrm{Be}-$ accretion, accretion disks open clusters and associations: individual: $\eta$ Chamealeontis - protoplanetary disks

\section{Introduction}

The evolution of protoplanetary disks affects planet formation and migration. Statistical studies of both excess infrared and $\mathrm{H} \alpha$ emission demonstrate that the majority of disks dissipate within 2-3 Myr after the collapse of the parent molecular cloud (e.g., Haisch et al. 2001; Fedele et al. 2010). This timescale sets a limit on the time available to build up the atmospheres of gas giant planets. The evolution and dissipation of disks differ from system to system; a small fraction of stars retain their gas and dust disks for 5-10 Myr.

One such cluster, the $\eta$ Chamaeleontis association (Mamajek et al. 1999b), is located at a distance of $d=94 \mathrm{pc}^{1}$ (van Leeuwen 2007; see discussion in Murphy et al. 2010) with an age of 4-10 Myr (e.g., Lawson et al. 2001; Lawson \& Feigelson 2001; Luhman \& Steeghs 2004; Herczeg \& Hillenbrand 2015). The total disk fraction of the association is in the range of $35-45 \%$ if stars of all masses are included ${ }^{2}$. Of the 15 canonical low-mass members, 8 retain dust disks as identified from excess IR emission (Megeath et al. 2005; Sicilia-Aguilar et al. 2009), thereby offering a unique opportunity to study disk and stellar properties at stages when giant planet formation should be coming to an end.

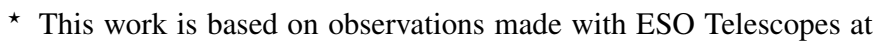
the Paranal Observatory under program ID 084.C-1095.

1 Recent parallax measurements with Gaia give distances of 94 and $102 \mathrm{pc}$ for HD 75505 and RECX 1, respectively (Gaia Collaboration 2016), in good agreement with previous estimates. In this paper we adopt a distance of $94 \mathrm{pc}$.

2 The lower bound also takes into account seven recent $\eta$ Cha cluster member candidates (Murphy et al. 2010; Lopez Martí et al. 2013), one of which has been confirmed to have a disk (Simon et al. 2012).
}

In the viscous accretion model of disk evolution (e.g., Hartmann et al. 1998), the accretion rate is expected to decrease with age. The accretion properties of old disks, including those in the $\eta$ Chamaeleontis association, are therefore important for comparison to the accretion properties of younger systems. However, these measurements may be complicated because emission from young stars include photospheric and accretion components and are affected by extinction, all of which may vary with time (e.g., Bertout et al. 1988; Basri \& Bertout 1989; Gullbring et al. 1998; Sicilia-Aguilar et al. 2010). Recent improvements in evaluating photospheric and accretion properties of young stars have been driven by simultaneous fits of spectral type, extinction, and accretion luminosity to broadband optical spectra, using young stars as templates (e.g., Manara et al. 2013a, 2016, 2017; Herczeg \& Hillenbrand 2014).

In this paper, we analyze flux-calibrated X-shooter optical spectra of 15 low-mass members of the $\eta$ Cha cluster to measure accretion and photospheric properties of stars in the association. The paper is structured as follows: after describing the observational setup in Sect. 2, in Sect. 3 we determine the stellar parameters and investigate each star for extinction and continuum excess; in Sect. 4 we investigate each sample member for accretion and infer the accretion luminosity of the accreting pre-main sequences (PMS); in Sect. 5 we present hydrogen emission lines and the derived mass accretion rates; in Sect. 6 we discuss the results, and summarize the study in Sect. 7.

\section{Observations and data reduction}

As part of a survey on T Tauri stars in the $\eta$ Cha association and Chamaeleon I and II (Manara et al. 2016; Program ID 084.C1095, PI: Herczeg), a subsample of $\eta$ Cha cluster members have 
Table 1. Observation log of VLT/X-shooter observations in the $\eta$ Cha association.

\begin{tabular}{|c|c|c|c|c|c|c|c|}
\hline Object & $\mathrm{RA}(\mathrm{J} 2000)$ & $\operatorname{Dec}(\mathrm{J} 2000)$ & $\begin{array}{r}\text { Obs. date } \\
\text { [UTC] }\end{array}$ & Mode & $\begin{array}{r}\text { Exp. (UVB) } \\
{[\mathrm{s}]}\end{array}$ & $\begin{array}{r}\text { Exp. (VIS) } \\
{[\mathrm{s}]}\end{array}$ & $\begin{array}{r}\text { Exp. (NIR) } \\
{[\mathrm{s}]}\end{array}$ \\
\hline ECHA J0836.2-7908 (J0836) & 83610.27 & -790817.65 & 2010-01-20 & NOD & 800 & 790 & 780 \\
\hline RECX 1 & 83655.77 & -785645.71 & 2010-01-18 & NOD & 48 & 20 & 40 \\
\hline ECHA J0838.9-7916 (J0838) & 83850.65 & -791613.66 & 2010-01-18 & NOD & 200 & 180 & 200 \\
\hline ECHA J0841.5-7853 (J0841) & 84129.24 & -785303.45 & 2010-01-19 & NOD & 360 & 388 & 388 \\
\hline RECX 3 & 84136.46 & -790327.97 & 2010-01-20 & NOD & 220 & 240 & 240 \\
\hline RECX 4 & 84223.07 & -790400.60 & 2010-01-18 & NOD & 100 & 90 & 80 \\
\hline RECX 5 & 84226.37 & -785744.48 & 2010-01-19 & NOD & 120 & 96 & 120 \\
\hline RECX 6 & 84238.71 & -785441.97 & 2010-01-20 & NOD & 170 & 180 & 180 \\
\hline RECX 7 & 84307.19 & -790450.84 & 2010-01-20 & STARE & 55 & 60 & 60 \\
\hline ECHA J0843.3-7915 (J0843) & 84317.24 & -790516.74 & $2010-01-18$ & NOD & 100 & 90 & 100 \\
\hline ECHA J0844.2-7833 (J0844) & 84408.61 & -783345.25 & $2010-01-18$ & NOD & 460 & 480 & 460 \\
\hline RECX 9 & 84415.65 & -785905.43 & 2010-01-19 & NOD & 240 & 60 & 240 \\
\hline RECX 10 & 84430.81 & -784629.02 & 2010-01-19 & NOD & 100 & 90 & 100 \\
\hline RECX 11 & 84701.25 & -785934.02 & 2010-01-18 & NOD & 50 & 30 & 40 \\
\hline RECX 12 & 84755.72 & -785452.74 & 2010-01-19 & NOD & 100 & 120 & 120 \\
\hline
\end{tabular}

Notes. Column 1 lists the names of all objects in the observed sample. Abbreviations used in this work are given in parentheses. The observations were carried out in "NODDING" (NOD) or "STARE" mode with the 1 " $\times 11^{\prime \prime}$ slit in the UVB arm and 0". $\times 11^{\prime \prime}$ slits in both VIS and NIR arm. The exposure time (Exp.) for each arm is given in the last three columns. Complementary broad slit observations in "STARE" mode used the $5^{\prime \prime} \times 11^{\prime \prime}$ slits for UVB and VIS arm, and the 1".5 × 11" slit for the NIR arm (except for targets J0836, RECX 3, RECX 6, and RECX 7, for which a slit of $5^{\prime \prime} \times 11^{\prime \prime}$ was used in the NIR arm).

been observed with the ESO/VLT X-shooter echelle spectrograph over three nights in January 2010 (Table 1). The X-shooter sample consists of 15 low-mass stars among the cluster members found by, e.g., Luhman \& Steeghs (2004). Four new probable cluster members and three potential members were identified by Murphy et al. (2010) in the outskirts of the cluster after the observations presented here had been conducted and are therefore not included in our sample.

The X-shooter spectrograph covers a broad wavelength range, from 300 to $2500 \mathrm{~nm}$ using three different arms (UVB: $\lambda \lambda$ 300-550 nm, VIS: $\lambda \lambda$ 550-1000 nm, NIR: $\lambda \lambda 1000-2500 \mathrm{~nm}$, (Vernet et al. 2011)). To optimize the flux calibration, we combined short (3-65 s) broad-slit (1".5-5'.0) and deep (20-800 s) narrow-slit $\left(0 \prime \prime 4-1{ }^{\prime \prime} 0\right)$ observations. The observation log is given in Table 1. In this paper we present the optical spectra extracted from the UVB and VIS arms.

Data reduction, carried out with the $\mathrm{X}$-shooter pipeline XSH 1.2.0 (Modigliani et al. 2010), consisted of bias and flatfield corrections, combination of single frames obtained in the "NODDING" mode, wavelength calibration, rectifying each order, and then merging the orders. The extraction of the spectra and the sky removal were performed with the IRAF ${ }^{3}$ task apall.

The response function was measured using the spectrophotometric standard star GD 71 (Hamuy et al. 1994; Vernet et al. 2009) observed at the beginning of each night. Since the comparison of the response functions from the individual nights only yielded small differences, all UVB-arm observations were calibrated with the response function derived from the first night and all VIS-arm observations with an average response function of all three nights.

In order to account for wavelength-dependent slit losses, narrow-slit observations are scaled to the broad-slit observations with low order polynomials fitted to spectral regions with

\footnotetext{
3 IRAF is distributed by the National Optical Astronomy Observatories, which are operated by the association of Universities for Research in Astronomy, Inc, under cooperative agreement with the National Science Foundation.
}

high signal-to-noise ratio. The stability and accuracy of the flux calibration is estimated by comparing observations of telluric standards across all nights. Figure 1 shows the comparison of multi-epoch X-shooter spectra of three standards stars with optical photometry: $B_{T}, V_{T}$ band photometry from the Tycho-2 cat$\operatorname{alog}^{4}$ and $R$ band from the NOMAD catalog 5 . In general, the observed spectra agree well with the photometry; the fluxes at $B, V$, and $R$-band wavelengths are located within the error bars of the photometry and there is an overall scatter in all standards of $\sim 4 \%$. Based on this comparison the flux calibration accuracy is $\sim 4 \%$.

\section{Stellar parameters}

Measurements of stellar parameters for low-mass PMS stars may be contaminated by the accretion continuum, which if not considered can lead to degeneracies between spectral type, extinction, and accretion measurements. These degeneracies are minimized when measuring stellar parameters from flux-calibrated optical spectra that cover a wide wavelength range (e.g., Manara et al. 2013a; Herczeg \& Hillenbrand 2014; Alcalá et al. 2014). In this section we first estimate spectral type (SpT) and veiling following Herczeg \& Hillenbrand (2014). Based on this initial estimate, we then perform a grid comparison of the program stars with stellar templates of different SpT, and different veiling and extinction values. Finally, the physical stellar properties (effective temperature $\left(T_{\text {eff }}\right)$, luminosity $\left(L_{*}\right)$, radius $\left(R_{*}\right)$, and mass $\left(M_{*}\right)$ ) are inferred using tabulated conversions, and from comparison with evolutionary tracks on the HertzsprungRussell diagram.

\footnotetext{
4 http://cdsarc.u-strasbg.fr/viz-bin/Cat?I/259; converted to Johnson $B$ - and $V$-band photometry.

5 http://cdsarc.u-strasbg.fr/viz-bin/Cat?I/297; $R$-band photometry for HIP 40415 from the USNO-B1.0 catalog, http://cdsarc.u-strasbg.fr/viz-bin/Cat?I/284
} 
M. Rugel et al.: X-shooter observations of low-mass stars in the $\eta$ Chamaeleontis association

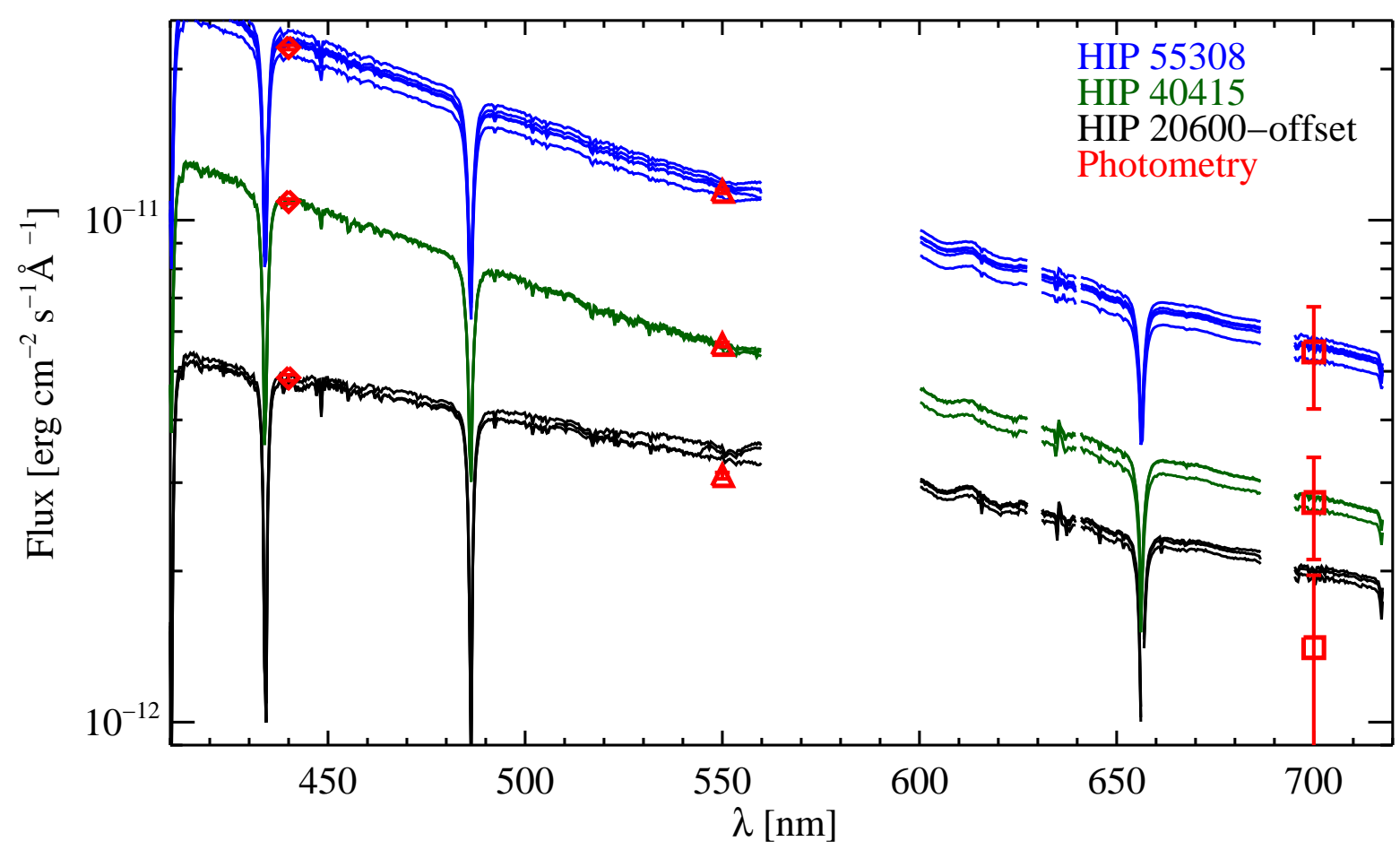

Fig. 1. Comparison of telluric standards HIP 55308 (blue), HIP 40415 (green) and HIP 20600 (black), observed in different epochs (multiple instances of one color), with literature photometry (red) in the $B$-band (diamonds), $V$-band (triangles), and $R$-band (squares). The spectra of HIP 20600 and the corresponding photometry are displayed with a constant offset of $1 \times 10^{-12} \mathrm{erg} \mathrm{s}^{-1} \mathrm{~cm}^{-2} \AA^{-1}$.

Table 2. Spectral types derived from spectral indices and adopted values after comparison to non-accreting PMS.

\begin{tabular}{lcccccc}
\hline \hline Object & $\begin{array}{c}\text { R5150 } \\
\text { K0-M0 })\end{array}$ & $\begin{array}{c}\text { TiO6800 } \\
(\text { K5-M0.5 })\end{array}$ & $\begin{array}{c}\text { TiO7140 } \\
\text { (M0-M4.5) }\end{array}$ & $\begin{array}{c}\text { TiO8465 } \\
(\text { M4-M8) }\end{array}$ & $\begin{array}{c}\text { JTiO7140 } \\
\text { (K5-M7) }\end{array}$ & Adopted \\
\hline J0836 & - & - & - & M5.70 & M6.4 & M5.5 \\
RECX 1 & K5.7 & K5.4 & - & - & K6.4 & K6.0 \\
J0838 & - & - & - & M5.3 & M5.9 & M5.5 \\
J0841 & - & - & - & M5.1 & M5.6 & M5.0 \\
RECX 3 & - & - & M3.60 & - & M3.7 & M3.5 \\
RECX 4 & - & - & M1.7 & - & M1.3 & M1.5 \\
RECX 5 & - & - & M4.5 & M4.2 & M4.5 & M4.5 \\
RECX 6 & - & - & M3.1 & - & M3.2 & M3.0 \\
RECX 7 & K5.6 & K6.0 & - & - & K6.9 & K6.0 \\
J0843 & - & - & M3.6 & - & M3.7 & M4.0 \\
J0844 & - & - & - & M6.1 & M5.5 & M6.0 \\
RECX 9 & - & - & - & M4.7 & M5.2 & M4.5 \\
RECX 10 & - & K8.4 & M0.7 & - & M0.2 & M0.0 \\
RECX 11 & K5.7 & K5.2 & - & - & K6.0 & K6.0 \\
RECX 12 & - & - & M3.0 & - & M3.1 & M3.0 \\
\hline
\end{tabular}

Notes. Columns 2-5 give spectral types from indices by Herczeg \& Hillenbrand (2014). The index JTiO 7140 is listed for comparison (Jeffries et al. 2007); however, it is not used in the final classification. The validity range of each spectral index is indicated in parentheses. The last column shows the adopted spectral type in this work (see Sects. 3.1 and 3.3 for details).

\subsection{Spectral types}

Our initial spectral types are calculated from atomic absorption and molecular bands, quantified in the spectral indices R5150, TiO6800, TiO7140, and TiO8465 that were developed for young stars (Herczeg \& Hillenbrand 2014). The indices TiO6800, TiO7140, and TiO8465 compare integrated flux within the absorption and the continuum band. For R5150, a linear fit over low and high wavelength continuum regions is used to estimate the continuum on the band. In two cases, J0843 and J0844, these indices are corrected for veiling (see Sect. 3.2). These spectral types agree with those that would have been measured from the TiO7140 index of Jeffries et al. (2007).
Table 2 lists the spectral type obtained from each index, which combine to form an initial estimate of spectral type. The final spectral types are adopted in Sect. 3.3 by comparing the spectra to a grid of spectra of non-accreting young stars.

\subsection{Veiling estimates}

Veiling describes the change in the depth of photospheric absorption lines due to excess continuum emission (e.g., Basri \& Batalha 1990). Veiling at blue wavelengths is measured in the gravity-sensitive $\mathrm{Ca}$ I $\lambda 422.7 \mathrm{~nm}$ absorption line, following the approach of Herczeg \& Hillenbrand (2014) but adapted to 


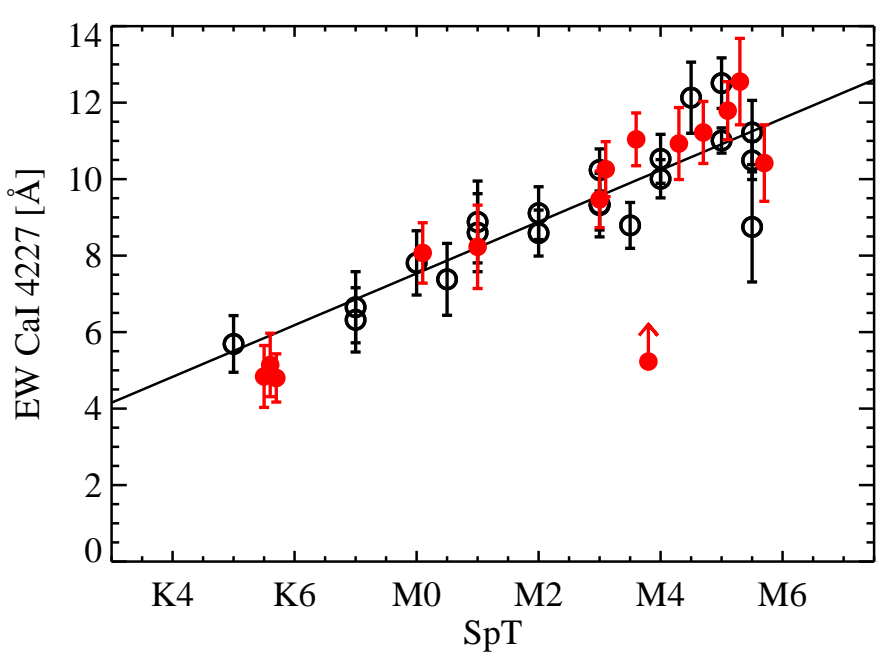

Fig. 2. Equivalent width of the Ca I $\lambda 422.7 \mathrm{~nm}$ line vs. spectral type. Red filled circles denote observations presented in this work, black empty circles are from template stars (Manara et al. 2013b). Due to strong emission lines inside the absorption feature, we determine a lower limit for J0843 (red arrow). The black line denotes the relation between the equivalent widths and spectral types of non-accreting stars (Eq. (1)).

higher spectral resolution using the non-accreting photospheric templates from Manara et al. (2013b). For templates, the relationship between spectral type and equivalent width (Fig. 2) is described by

$E W_{\text {CaI }} \lambda 422.7 \mathrm{~nm}=-31.7+0.676 \times \mathrm{SpT}$,

where $\mathrm{SpT}$ is spectral type and a spectral type of M0 is equivalent to $\mathrm{SpT}=58$. Figure 2 shows that most $\eta$-Cha-cluster objects have equivalent widths consistent with those expected from the templates. These objects are assumed to have negligible veiling.

Two objects, J0843 and J0844, show significant veiling. The Ca I $\lambda 422.7 \mathrm{~nm}$ absorption feature in J0843 is detected but blended with emission lines. Assuming that the absorption profile is Gaussian with a similar width to that of the template spectra, the lower limit on the Ca I equivalent width is estimated to be at $5.2 \AA$ (Fig. 3), implying a veiling of $r_{422.7 \mathrm{~nm}} \lesssim 1$ (Eq. (1) $)^{6}$. The absorption feature is not detected in J0844 (Fig. 3), indicating that the veiling is high.

\subsection{Grid comparison with PMS stellar templates}

In order to verify the spectral type and veiling, we compare each spectrum to a set of PMS, non-accreting stellar templates obtained from the X-shooter library of young stars (Manara et al. 2013b). We model veiling and extinction simultaneously by adding constant accretion continuum flux to the template (Herczeg \& Hillenbrand 2014), and by convolving each model with the extinction curve of Cardelli et al. (1989), with a total-to-selective extinction ratio of $R_{V}=3.1$. The best-fit parameters are initially determined from spectral fits and are then tweaked manually to obtain the best visual representation of the spectrum.

All objects without veiling in the $\mathrm{Ca}$ I $\lambda 422.7 \mathrm{~nm}$ absorption feature (see Sect. 3.2) are best modeled without adding excess flux longward of $400 \mathrm{~nm}$. All sources, including those with ongoing accretion, are also well fit with no reddening, consistent with previous studies (e.g., Luhman \& Steeghs 2004).

\footnotetext{
6 The amount of veiling at wavelength $\lambda$ is given by $r_{\lambda}=f_{\text {excess }} / f_{\text {phot }}$, where $f_{\text {excess }}(\lambda)$ is flux in excess to the flux of the photosphere, $f_{\text {phot }}(\lambda)$.
}

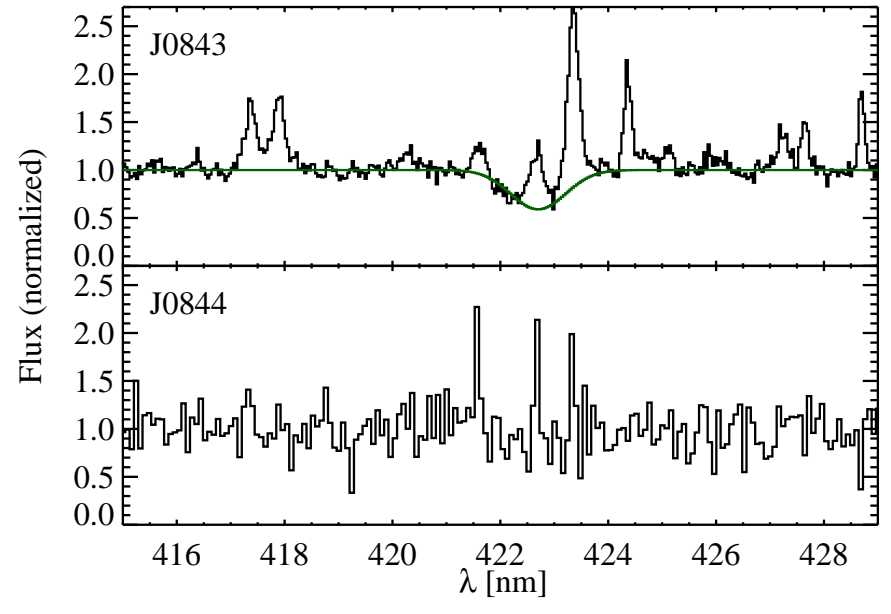

Fig. 3. Continuum-normalized spectrum around the $\mathrm{Ca}$ I $\lambda 422.7 \mathrm{~nm}$ absorption line (black) for the veiled stars J0843 (top) and J0844 (bottom). The absorption line from J0843 is contaminated by emission in the same transition. The equivalent width, and subsequently the veiling, are measured by fitting a Gaussian profile (green) to the spectrum, avoiding regions with emission. The absorption line from J0844 is not detected.

An iterative approach is used for the two objects with strong veiling in the CaI $\lambda 422.7$ line, J0843 and J0844. For the final models, the extinction is fixed at $A_{V}=0$ mag. The templates are fixed to the PMS stars TWA 9B (TW Hya association, $\mathrm{SpT}=\mathrm{M} 3)$ and $\mathrm{SO} 925(\sigma$ Ori region, $\mathrm{SpT}=\mathrm{M} 5.5$; Manara et al. 2013b). The best-fit value for the veiling at $751 \mathrm{~nm}$ is $r_{751}=0.05$ in both objects. The veiling translates to excess fluxes of $f_{\text {excess }}=9.1 \times 10^{-16} \mathrm{erg} \mathrm{s}^{-1} \mathrm{~cm}^{-2} \AA^{-1}$ for $\mathrm{J} 0843$ and $f_{\text {excess }}=6.9 \times 10^{-17} \mathrm{erg} \mathrm{s}^{-1} \mathrm{~cm}^{-2} \AA^{-1}$ for J0844. After accounting for this veiling, the spectral type from spectral indices changes to M4.0 for J0843 and stays at M6.0 for J0844.

The final spectral types are listed to a precision of one subclass for stars earlier than $\mathrm{M} 0$ and to 0.5 subclasses for stars later than M0, adopting the spacing of the sample of non-accreting template stars (see also Manara et al. 2013b).

\subsection{Effective temperature, luminosity, and stellar radius}

We derive effective temperatures from spectral types using the conversion table proposed by Herczeg \& Hillenbrand (2014) (see also Pecaut \& Mamajek 2013), as estimated by comparing the optical spectra of young templates to the recent BT-Settl models of synthetic spectra (Allard et al. 2012).

Stellar luminosities are obtained from the continuum flux at $751 \mathrm{~nm}$ and using the bolometric flux conversion table from Herczeg \& Hillenbrand (2014). For J0843 and J0844, the estimated flux in the accretion continuum at $751 \mathrm{~nm}$ (see Sect. 3.3) is subtracted before calculating $L_{*}$. The stellar radius is then calculated from the Stefan Boltzmann law, $L_{*}=4 \pi R_{*}^{2} \sigma T_{\mathrm{eff}}^{4}$. The results are listed in Table 3.

In Table 4 we compare the derived stellar parameters with previous classifications of the $\eta$ Cha association (Luhman \& Steeghs 2004; Lyo et al. 2004). The spectral types agree well within the uncertainties. Consistent with the errors of the classification, there is a trend of spectral types in this work being slightly earlier for early $M$ stars and later for late $M$ stars. The effective temperatures are systematically colder for cold objects, which is due to the different SpT- $T_{\text {eff }}$ conversions used in this work. Use of the Pecaut \& Mamajek (2013) SpT- $T_{\text {eff }}$ scale would have led to even lower temperatures for these stars. 
Table 3. Derived stellar parameters in the $\eta$ Cha association.

\begin{tabular}{lcccccc}
\hline \hline Object & $\begin{array}{c}T_{\text {eff }} \\
{[\mathrm{K}]}\end{array}$ & $\begin{array}{c}f_{751} \\
{\left[1 \times 10^{-13} \mathrm{erg} \mathrm{s}^{-1} \mathrm{~cm}^{-2} \AA^{-1}\right]}\end{array}$ & $\begin{array}{c}L_{*} \\
{\left[L_{\odot}\right]}\end{array}$ & $\begin{array}{c}R_{*} \\
{\left[R_{\odot}\right]}\end{array}$ & $\begin{array}{c}M_{*} \\
{\left[M_{\odot}\right]}\end{array}$ & Infrared Class $^{1}$ \\
\hline J0836 & 2920 & 0.038 & 0.019 & 0.54 & 0.069 & Class III \\
RECX1 & 4115 & 3.100 & 0.980 & 1.90 & 0.750 & Class III \\
J0838 & 2920 & 0.066 & 0.034 & 0.72 & 0.075 & Class III \\
J0841 & 2980 & 0.043 & 0.021 & 0.54 & 0.086 & TO/flat \\
RECX3 & 3300 & 0.280 & 0.100 & 0.98 & 0.250 & TO \\
RECX4 & 3640 & 0.620 & 0.200 & 1.10 & 0.460 & TO \\
RECX5 & 3085 & 0.150 & 0.067 & 0.91 & 0.150 & TO \\
RECX6 & 3410 & 0.300 & 0.100 & 0.93 & 0.320 & Class III \\
RECX7 & 4115 & 2.200 & 0.700 & 1.60 & 0.780 & Class III \\
J0843 & 3190 & 0.180 & 0.073 & 0.88 & 0.200 & Class II \\
J0844 & 2860 & 0.014 & 0.008 & 0.36 & 0.052 & flat \\
RECX9 & 3085 & 0.220 & 0.095 & 1.10 & 0.150 & TO \\
RECX10 & 3900 & 0.670 & 0.210 & 1.00 & 0.690 & Class III \\
RECX11 & 4115 & 1.500 & 0.460 & 1.30 & 0.830 & Class II \\
RECX12 & 3410 & 0.680 & 0.230 & 1.40 & 0.290 & Class III \\
\hline
\end{tabular}

References. ${ }^{(1)}$ Infrared disk classifications, as in Sicilia-Aguilar et al. (2009).

Table 4. Comparison of stellar parameters from the literature.

\begin{tabular}{lrrrrrrrr}
\hline \hline Object & $\begin{array}{r}\text { SpT } \\
\left(\text { Luhman }^{1}\right)\end{array}$ & $\begin{array}{r}\text { SpT } \\
\left(\text { Lyo }^{2}\right)\end{array}$ & $\begin{array}{r}\text { SpT } \\
\text { (this work) }\end{array}$ & $\begin{array}{r}T_{\text {eff }}[\mathrm{K}] \\
\left(\text { Luhman }^{1}\right)\end{array}$ & $\begin{array}{r}T_{\text {eff }}[\mathrm{K}] \\
\text { (this work) }\end{array}$ & $\begin{array}{r}L_{*}\left[L_{\odot}\right] \\
\left(2 \text { MASS-J }^{3}\right)\end{array}$ & $\begin{array}{r}L_{*}\left[L_{\odot}\right] \\
\left(\text { Lyo }^{2}\right)\end{array}$ & $\begin{array}{r}L_{*}\left[L_{\odot}\right] \\
\text { (this work) }\end{array}$ \\
\hline J0836 & M5.5 & M5.3 & M5.5 & 3058 & 2920 & 0.020 & 0.020 & 0.0190 \\
RECX1 & K6 & K7.0 & K6.0 & 4205 & 4115 & 0.880 & 1.000 & 0.9800 \\
J0838 & M5.25 & M5.0 & M5.5 & 3091 & 2920 & 0.034 & 0.035 & 0.0340 \\
J0841 & M4.75 & M4.7 & M5.0 & 3161 & 2980 & 0.021 & 0.023 & 0.0210 \\
RECX3 & M3.25 & M3.0 & M3.5 & 3379 & 3300 & 0.089 & 0.097 & 0.1000 \\
RECX4 & M1.75 & M1.3 & M1.5 & 3596 & 3640 & 0.210 & 0.240 & 0.2000 \\
RECX5 & M4 & M3.8 & M4.5 & 3270 & 3085 & 0.056 & 0.062 & 0.0670 \\
RECX6 & M3 & M3.0 & M3.0 & 3415 & 3410 & 0.100 & 0.110 & 0.1000 \\
RECX7 & K6 & K6.9 & K6.0 & 4205 & 4115 & 0.690 & 0.790 & 0.7000 \\
J0843 & M3.25 & M3.4 & M4.0 & 3379 & 3190 & 0.074 & 0.083 & 0.0730 \\
J0844 & M5.75 & M5.5 & M6.0 & 3024 & 2860 & 0.011 & 0.010 & 0.0078 \\
RECX9 & M4.5 & M4.4 & M4.5 & 3198 & 3085 & 0.090 & 0.096 & 0.0950 \\
RECX10 & M1 & M0.3 & M0.0 & 3705 & 3900 & 0.210 & 0.230 & 0.2100 \\
RECX11 & K5.5 & K6.5 & K6.0 & 4278 & 4115 & 0.530 & 0.590 & 0.4600 \\
RECX12 & M3.25 & M3.2 & M3.0 & 3379 & 3410 & 0.240 & 0.250 & 0.2300 \\
\hline
\end{tabular}

References. ${ }^{(1)}$ Luhman \& Steeghs (2004); ${ }^{(2)}$ Lyo et al. (2004); ${ }^{(3)}$ Cutri et al. (2012), Herczeg \& Hillenbrand (2015).

Measured stellar luminosities agree well within the errors (see Table 4) to previous measurements (Lyo et al. 2004) and to luminosities inferred from $J$-Band (Cutri et al. 2012; using bolometric corrections from either Pecaut \& Mamajek 2013 or Herczeg \& Hillenbrand 2015).

\subsection{The Hertzsprung-Russell diagram}

We use stellar isochrones from Baraffe et al. (2015) to infer stellar ages and masses. The isochrones are first mapped to a finer grid in $T_{\text {eff }}$. For a given grid- $T_{\text {eff }}$ value, the isochrones are interpolated onto a finer age grid. The adopted stellar mass is given by the closest grid value in $T_{\text {eff }}$ and $L_{*}$.

Figure 4 shows the sample overplotted with evolutionary tracks from Baraffe et al. (2015). A large fraction of the sample (2/3) lies between the $1 \mathrm{Myr}$ and $5 \mathrm{Myr}$ isochrones, approximately following the trend of the isochrones. The known binaries are located above the single stars. The median age of single stars hotter than $3300 \mathrm{~K}$ is $5 \mathrm{Myr}$. This agrees well with some of the previous results (Luhman \& Steeghs 2004; Herczeg \& Hillenbrand 2015) and place the $\eta$ Cha cluster at a significantly younger age than the others (e.g., $11 \pm 3 \mathrm{Myr}$, Bell et al. 2015). As a general caveat, masses and ages inferred from Hertzsprung-Russell diagrams may be misleading for lowmass stars when spectra are fit with single-temperature photospheres (e.g., Gully-Santiago et al. 2017).

\section{Mass accretion rate}

In this section, we measure accretion rates and upper limits of our sample from UVB-arm spectra of X-shooter. Section 4.1 discusses the Balmer jump as a diagnostic of excess continuum emission produced by ongoing accretion. The excess flux is quantified by modeling the observed spectrum, as described in Sect. 4.2, and used to derive the mass accretion rate. The model consists of non-accreting PMS spectra as proxies for photospheric emission of young stars and the emission of a planeparallel slab as a proxy for the radiation from regions heated in the accreting process. While the emission from the plane-parallel 


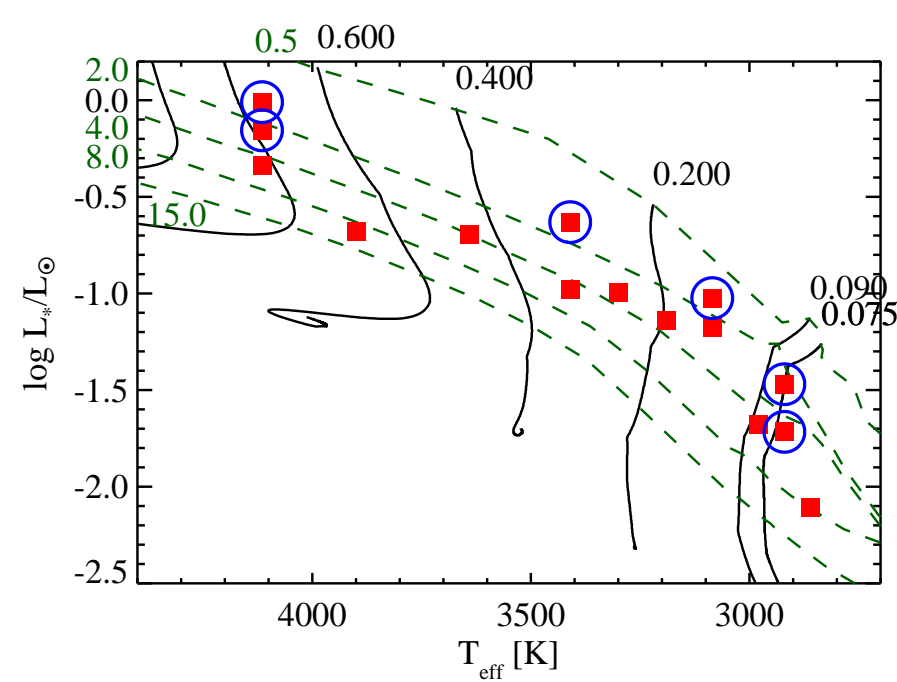

Fig. 4. Hertzsprung-Russell diagram of the $\eta$ Cha association. Evolutionary tracks (mass in $M_{\odot}$ ) are shown as black solid lines and isochrones (age in Myr) as green dashed lines (both Baraffe et al. 2015). $\eta$ Cha cluster stars are shown as red squares. Binary stars are encircled in blue (as in Table 1 of Sicilia-Aguilar et al. 2009).

hydrogen slab is not a physical model (for physical models see, e.g., Calvet \& Gullbring 1998; Ingleby et al. 2013), the accretion spectrum provides a reasonable fit to the continuum emission and a reasonable bolometric correction to account for emission outside of the observed spectral range. Individual objects are discussed in Sect. 4.3.

\subsection{The Balmer jump}

The presence of excess Balmer-continuum emission produced by accretion is quantified here by measuring the Balmer jump, defined as the flux ratio $f(360 \mathrm{~nm}) / f(400 \mathrm{~nm})^{7}$. The X-shooter UVB spectra are shown in Figs. 6 and 7 along with template nonaccreting PMS stars (Manara et al. 2013b; Stelzer et al. 2013), with results on the Balmer jump shown in Fig. 5. The dashed line $(f(360 \mathrm{~nm}) / f(400 \mathrm{~nm})=0.5)$ indicates the threshold between accreting and non-accreting sources (Herczeg et al. 2009) consistent with the non-accreting templates of Manara et al. (2013b).

Of the $\eta$ Cha cluster members, seven sources show a Balmer jump above this threshold. Three sources have strong UV-excess emission (RECX 5: Balmer jump 0.8, J0843: 2.1, and J0844: 2.6), while four have a moderate excess (0.5-0.6: RECX 11, RECX 12, RECX 9, and J0838). In the case of J0838, the continuum level at $360 \mathrm{~nm}$ is enhanced with respect to the template, while at wavelengths below $355 \mathrm{~nm}$ the spectrum agrees with the template within the noise (Fig. 6). The enhanced Balmer jump of this star is therefore not considered as an indicator of accretion. The UV-excess in RECX 12 is attributed to chromospheric activity as the $\mathrm{H} \alpha$ line is narrow and has a lower equivalent width than expected for mass accretion (see Sect. 5), and has a UV spectrum consistent with the non-accreting template TWA 15A (Fig. 6). RECX 12 is discussed in more detail in Sect. 5. The UV-excess is clearly visible in the spectra of RECX 9 and RECX 11.

\footnotetext{
7 The Balmer jump measured here includes the photosphere and is not a photosphere-subtracted measurement of the accretion continuum.
}

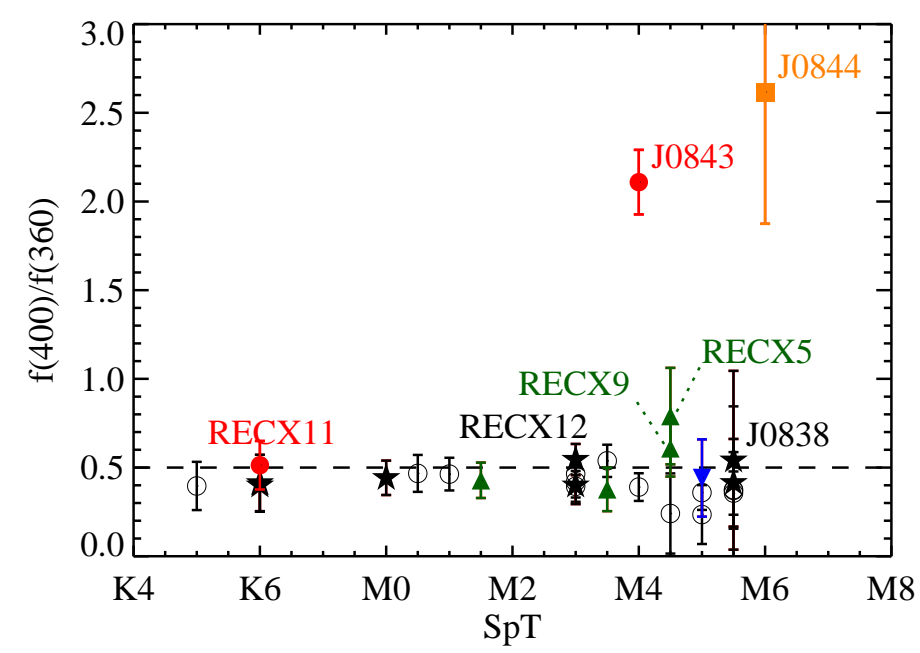

Fig. 5. Observed Balmer jump of the $\eta$ Cha cluster members (filled symbols) and non-accreting PMSs from Manara et al. (2013b), which were used as templates in this work (black empty circles). The black dashed line highlights an observed Balmer jump of 0.5. The shape and color of the filled symbols highlight the SED classifications from Sicilia-Aguilar et al. (2009): black stars denote Class III objects, red circles Class II objects, green triangles transitional objects, the blue inverted triangle the transitional/flat object J0841, and the orange box the flat source J0844.

Table 5. Parameter ranges of the hydrogen slab model.

\begin{tabular}{lrrr}
\hline \hline & Min. & Max. & Step size \\
\hline$T_{\text {eff }}[\mathrm{K}]$ & 6000 & 11000 & 500 \\
$\log \left(n_{\mathrm{H}}\left[\mathrm{cm}^{-3}\right]\right)$ & 12 & 15 & 0.01 \\
\hline
\end{tabular}

\subsection{Fitting the UV-excess}

The accretion continuum, modeled from a pure-hydrogen isothermal slab, is added to the photospheric template (see Sect. 3.3) to best fit the UV spectrum of the accreting star. The grid of slab models (Valenti et al. 1993) spans a parameter space in density and temperature of the hydrogen gas (Table 5), where the length of the slab is fixed to $l=2 \times 10^{7} \mathrm{~cm}$ (Herczeg et al. 2009). The bolometric correction is calculated by summing the accretion continuum luminosity over all wavelengths.

Best-fit parameters are calculated by minimizing a $\chi^{2}$-like function over multiple wavelength bands in the UVB arm of $\mathrm{X}$-shooter, with wavelengths selected to exclude sharp emission and absorption lines. The $\chi^{2}$-like minimization function is defined as $\chi_{\text {like }}^{2}=\sum_{\text {bands }}\left(\frac{\overline{f_{\text {obs }}}-\overline{f_{\text {model }}}}{\sigma\left(f_{\text {obs }}\right)}\right)$, where $\overline{f_{\text {obs }}}$ and $\overline{f_{\text {model }}}$ denote the median of each band in the observed spectrum and model, and $\sigma\left(f_{\text {obs }}\right)$ gives the standard deviation in each band in the observed spectrum. As also described in Manara et al. (2013a), this does not represent a true $\chi^{2}$ distribution as the noise in the template spectrum is neglected. The models are visually compared to confirm that the best-fit model matches the observed spectrum. The uncertainties are estimated from the range of UV-excess fluxes spanned by models that yield a similar $\chi^{2}$-like value as the best-fit model and match the spectrum visually (details are described in Sect. 4.3).

The measured UV-excess flux is converted to accretion luminosity $\left(L_{\text {acc }}\right)$. The mass accretion rates $(\dot{M})$ are then calculated by

$L_{\text {acc }}=\frac{G M_{*} \dot{M}}{R_{*}} \cdot\left(1-\frac{R_{*}}{R_{\text {trunc }}}\right)$, 


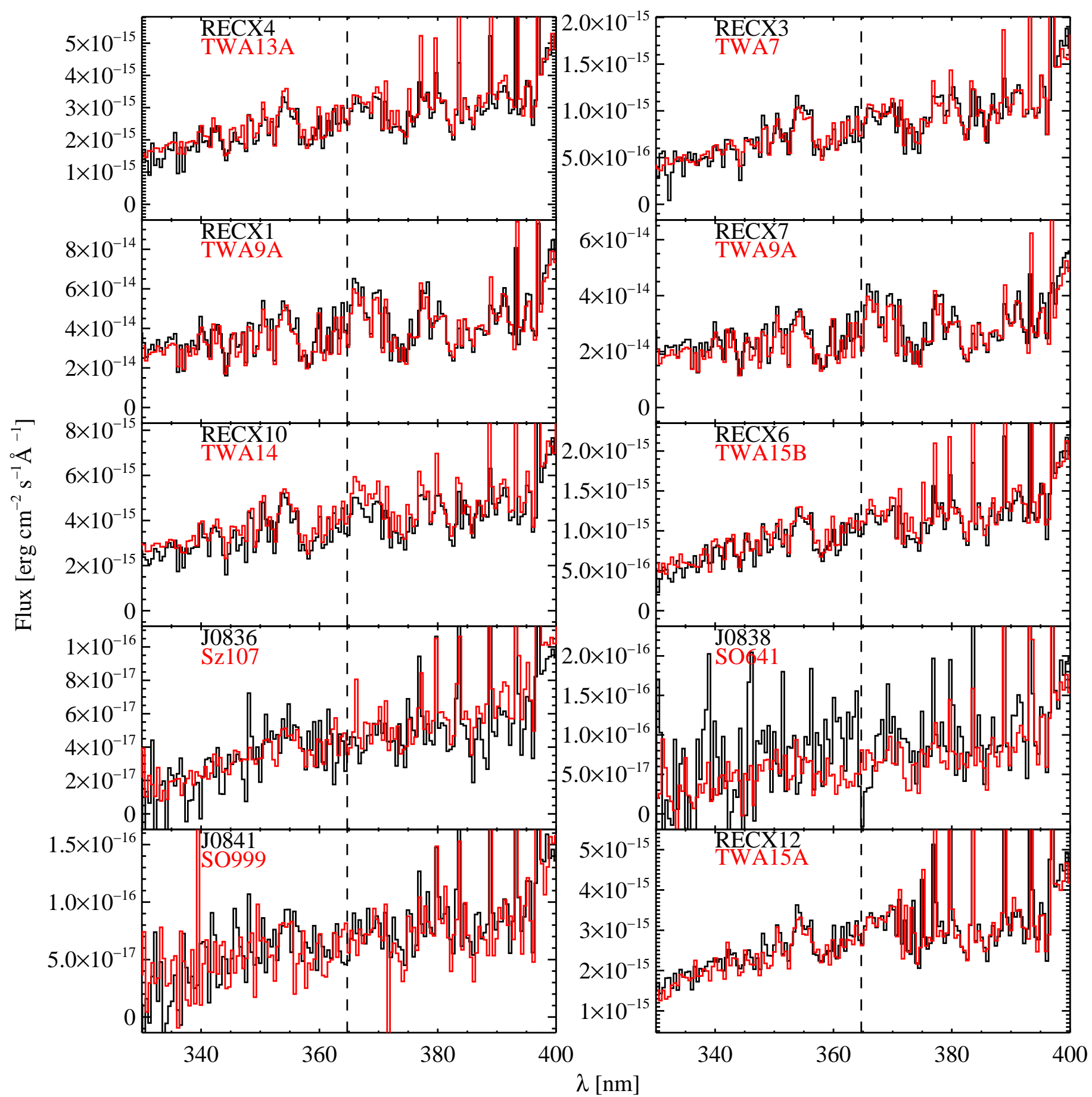

Fig. 6. Comparison of the UV spectrum for stars with an observed Balmer jump ratio typical for non-accreting stars (black), with template stars from Manara et al. (2013b) of similar spectral type (red; template names as in Manara et al. 2013b). The spectra are binned to $0.5 \mathrm{~nm}$ resolution and the templates are scaled to the target at $450 \mathrm{~nm}$. The dashed vertical line shows the theoretical location of the Balmer jump.

under the assumption that gas accretes from the disk truncation radius $R_{\text {trunc }}$ in free-fall onto the star, where $R_{\text {trunc }}=5 R_{*}$ is adopted for consistency with pre-existing measurements (see review by Hartmann et al. 2016).

\subsection{Description of fits and uncertainties}

Figures A.1-A.4 show the best-fit model for each object. The values of $L_{\text {acc }}$ and $\dot{M}$ are listed in Table 6.
Uncertainties in the accretion rates are introduced by uncertainties in the stellar parameters, the distance, and in the individual fits (see, e.g., Herczeg \& Hillenbrand 2008; Manara et al. 2013a). For our sample, uncertainties in $\dot{M}$ are $\sim 0.3$ dex. Weak accretors, such as RECX 11 , have uncertainties of $\sim 0.5$ dex because the excess is fainter than the photosphere, thereby increasing the uncertainty in bolometric correction.

Upper limits on $\dot{M}$ are difficult to estimate and depend on the ability to distinguish any excess emission from the underlying 
Table 6. Mass accretion properties in the $\eta$ Cha association.

\begin{tabular}{|c|c|c|c|c|c|c|c|c|}
\hline Object & $\begin{array}{r}E W_{\mathrm{H} \alpha \lambda} \lambda 656.3 \\
{[\AA]}\end{array}$ & $\begin{array}{r}L_{\mathrm{acc}, \mathrm{H} \alpha} \\
{\left[L_{\odot} / \mathrm{yr}\right]}\end{array}$ & $\begin{array}{r}\dot{M}_{\mathrm{H} \alpha} \\
{\left[M_{\odot} / \mathrm{yr}\right]}\end{array}$ & $\begin{array}{r}E W_{\mathrm{H} \beta \lambda} \lambda 486.1 \\
{[\AA]}\end{array}$ & $\begin{array}{r}L_{\mathrm{acc}, \mathrm{H} \beta} \\
{\left[L_{\odot} / \mathrm{yr}\right]}\end{array}$ & $\begin{array}{r}\dot{M}_{\mathrm{H} \beta} \\
{\left[M_{\odot} / \mathrm{yr}\right]}\end{array}$ & $\begin{array}{l}L_{\text {acc,cont }} \\
{\left[L_{\odot} / \mathrm{yr}\right]}\end{array}$ & $\begin{array}{r}\dot{M}_{\text {cont }} \\
{\left[M_{\odot} / \mathrm{yr}\right]}\end{array}$ \\
\hline 0836 & $-13.4 \pm 0.7$ & $24 \mathrm{e}-05$ & $<7.5 \mathrm{e}-12$ & $-11.1 \pm 0.4$ & $2.4 \mathrm{e}-05$ & $<7.6 \mathrm{e}-12$ & $<17 \mathrm{e}-05$ & $<5.3 \mathrm{e}-1$ \\
\hline & & & & & & & & \\
\hline & & & & $-10.4 \pm 0$ & & & & \\
\hline & & & & & & & & 12 \\
\hline & \pm 0.3 & $8 . / e-1$ & $<1.4 \mathrm{e}-1$ & \pm 0.1 & 4 & $<1.7 \mathrm{e}$ & 05 & -11 \\
\hline & $-4.2 \pm 0.3$ & $<4.9 \mathrm{e}-04$ & $<4.9 \mathrm{e}-11$ & $-2.7 \pm 0.2$ & $<7.3 \mathrm{e}-04$ & $<7.2 \mathrm{e}-11$ & $<1.7 \mathrm{e}$ & $<1.7 \mathrm{e}-11$ \\
\hline & $-13.5 \pm 0.7$ & $2.1 \mathrm{e}$ & $5.2 \mathrm{e}-1$ & $-14.7 \pm 0.4$ & $4.5 \mathrm{e}$ & $1.1 \mathrm{e}$ & & -10 \\
\hline & $5.3 \pm 0.2$ & $<2.2 \mathrm{e}-04$ & $<2.5 \mathrm{e}-1$ & $-4.0 \pm 0.1$ & $<3.4 \mathrm{e}$ & $<4.0$ & 05 & $<1.0 \mathrm{e}-11$ \\
\hline & \pm 0.1 & 4.3 & $<3$. & $<0.2$ & & $<8$. & & $<4.7$ \\
\hline & \pm 2.6 & & & $-42.6 \pm 0.7$ & & & & \\
\hline & \pm 5.5 & & & \pm 2.0 & & & & \\
\hline & $-12.6 \pm 0.5$ & & & $-10.7 \pm 0.3$ & & & & $1.2 \mathrm{e}-10$ \\
\hline & $-1.7 \pm 0.2$ & $<2.2 \mathrm{e}-04$ & $<1.3 \mathrm{e}-11$ & $-1.2 \pm 0.2$ & $<4.2 \mathrm{e}-04$ & $<2.5 \mathrm{e}-11$ & $<1.8 \mathrm{e}-04$ & $<1.1 \mathrm{e}-11$ \\
\hline & $-8.3 \pm 0.3$ & $3.9 \mathrm{e}-\mathrm{C}$ & & $-2.7 \pm 0.2$ & & & & $2.0 \mathrm{e}-10$ \\
\hline RECX12 & $-8.4 \pm 0.2$ & $<8.6 \mathrm{e}-04$ & $<1.6 \mathrm{e}-10$ & $-8.2 \pm 0.2$ & $<1.9 \mathrm{e}-03$ & $<3.6 \mathrm{e}-10$ & $<7.7 \mathrm{e}-04$ & $<1.5 \mathrm{e}-10$ \\
\hline
\end{tabular}

Notes. Columns 2-7 list equivalent widths (EW), accretion luminosity ( $L_{\text {acc}}$; using conversions from Alcalá et al. 2014), and mass accretion rates $(\dot{M})$ for $\mathrm{H} \alpha$ and $\mathrm{H} \beta$, respectively. The last two columns list $L_{\text {acc }}$ and $\dot{M}$ determined from $\mathrm{UV}$ excess.

photospheric spectrum and on an uncertain bolometric correction. In order to estimate upper limits of $\dot{M}$ for non-accreting objects, we perform a similar model fit as described above. The upper limits are compared to the detections in Fig. $8 \mathrm{a}$ in terms of the ratio of $L_{\text {acc }} / L_{*}$. For K stars, the upper limit of $L_{\text {acc }} / L_{*}$ for nonaccreting stars is similar to $L_{\text {acc }} / L_{*}$ of RECX 11 . For M stars, the upper limits are almost uniform at $\log L_{\mathrm{acc}} / L_{*} \approx-3.0$ (see also Manara et al. 2013b), with the exception of RECX 12 $\left(\log L_{\mathrm{acc}} / L_{*} \approx-2.7\right)$.

Comments on each fit to the spectra of accreting objects are provided below.

J0843 and J0844: due to the pronounced observed Balmer jump and strong excess in the Balmer and Paschen continua, the slab model parameters and therefore the $L_{\text {acc }}$ are well constrained. Models using templates within 0.5 spectral subclasses yield accretion rates that agree to within $10 \%$ of these values.

RECX 5: the best-fit $\dot{M}$ is calculated using the M4 template Sz 121. Model results using the M3 template TWA 9B and/or the M5 template Par-Lup3-2 also reproduce the UVB spectrum of RECX 5, and yield accretion rates that differ by $30 \%$ from the adopted best fit.

RECX 9: the best-fit $\dot{M}$ is calculated from the M4.5 template SO797. Model results using the M5 templates Par-Lup3-2 and SO641 yield accretion rates that differ by $45 \%$.

RECX 11: $\dot{M}$ of RECX 11 is uncertain by a factor of 2.5 because the UV excess is very small, so the slab model fit is not well constrained.

\section{Line emission}

Accretion and the related processes produce emission in many detectable lines, a subset of which is analyzed here: The $\mathrm{H} \alpha$ and $\mathrm{H} \beta$ transitions; the $\mathrm{He} \mathrm{I} \lambda 402.6$, HeI $\lambda 447.1$,

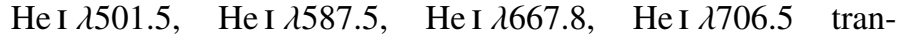
sitions and the blended He I and Fe I lines at $492.2 \mathrm{~nm}$
(He IFe I $\lambda$ 492.2, Alcalá et al. 2014); and the [O I] 1557.7 , [O I] $\lambda 630.0,[\mathrm{O}$ I] $\lambda 636.3$ forbidden transitions. The fluxes and equivalent widths of the detected lines are given in Tables 6 and B.1 to B.4.

Figures 10 and 11 show the $\mathrm{H} \alpha$ and $\mathrm{H} \beta$ profiles. The detection rate of the two transitions is $100 \%$ for $\mathrm{H} \alpha$ and $\sim 90 \%$ for $\mathrm{H} \beta$ throughout the full sample. For J0843, J0844, RECX 5, RECX 9, and RECX 11 , the $\mathrm{H} \alpha$ line profiles are very broad $\left(\Delta v>200 \mathrm{~km} \mathrm{~s}^{-1}\right)$ and slightly asymmetric, consistent with accretion. All other sources have narrow, symmetric $\mathrm{H} \alpha$ profiles with equivalent widths that are below the mass accretion threshold (White \& Basri 2003), consistent with a chromospheric origin of the line emission.

Transitions of He I are detected in J0844, J0843, RECX 5, RECX 9, and RECX 12 (see Tables B.1 to B.4). Emission in He I $\lambda 402.6$ is weakly detected in J0841. J0836 shows weak emission in the HeI $\lambda 447.1$ line. The emission lines for RECX 12 are weaker in all cases than for the accreting stars with detections in HeI. We note the following special line profiles: J0843 shows a broad, blueshifted component at lower flux than the central, narrow peak in the HeI $\lambda 447.1$ transition and broad wings in the He I $\lambda 587.5$ transition; the He I $\lambda 501.5$ line and $\mathrm{He} \mathrm{I}+\mathrm{Fe} \mathrm{I}$ blend at $\lambda 492.2$ are blended with Fe II emission at slightly higher wavelengths for J0844 and J0843; Fe II emission has also been found in heavily veiled objects, including some outbursts (e.g., Hessman et al. 1991; Hamann \& Persson 1992; van den Ancker et al. 2004; Fedele et al. 2007; Gahm et al. 2008).

The forbidden [O I] $\lambda 630.0 \mathrm{~nm}$ line, a diagnostic of winds (e.g., Hartigan et al. 1995; Simon et al. 2016), is detected from J0843 and J0841, and marginally $(<3 \sigma)$ from J0844 and RECX 5. To characterize velocity shifts of the line center, the wavelength solution was re-calibrated at the photospheric Li I $\lambda 670.8 \mathrm{~nm}$ line ${ }^{8}$. The [O I] $\lambda 630.0 \mathrm{~nm}$ line is blueshifted by $24 \mathrm{~km} \mathrm{~s}^{-1}$ for J0843, and not detectably shifted in J0841 (Fig. 9). Additionally, the [O I] $\lambda 557.7$ transition is detected from J0843, J0844, RECX 5, and RECX 9, while the [O I] $\lambda 636.3$ line is only detected from J0843.

\footnotetext{
8 The radial velocity of the two detected objects are measured at $11-13 \mathrm{~km} \mathrm{~s}^{-1}$ and are similar to the mean radial velocity of the $\eta$ Cha cluster $\left(14 \pm 1 \mathrm{~km} \mathrm{~s}^{-1}\right.$; e.g., Lopez Martí et al. 2013).
} 

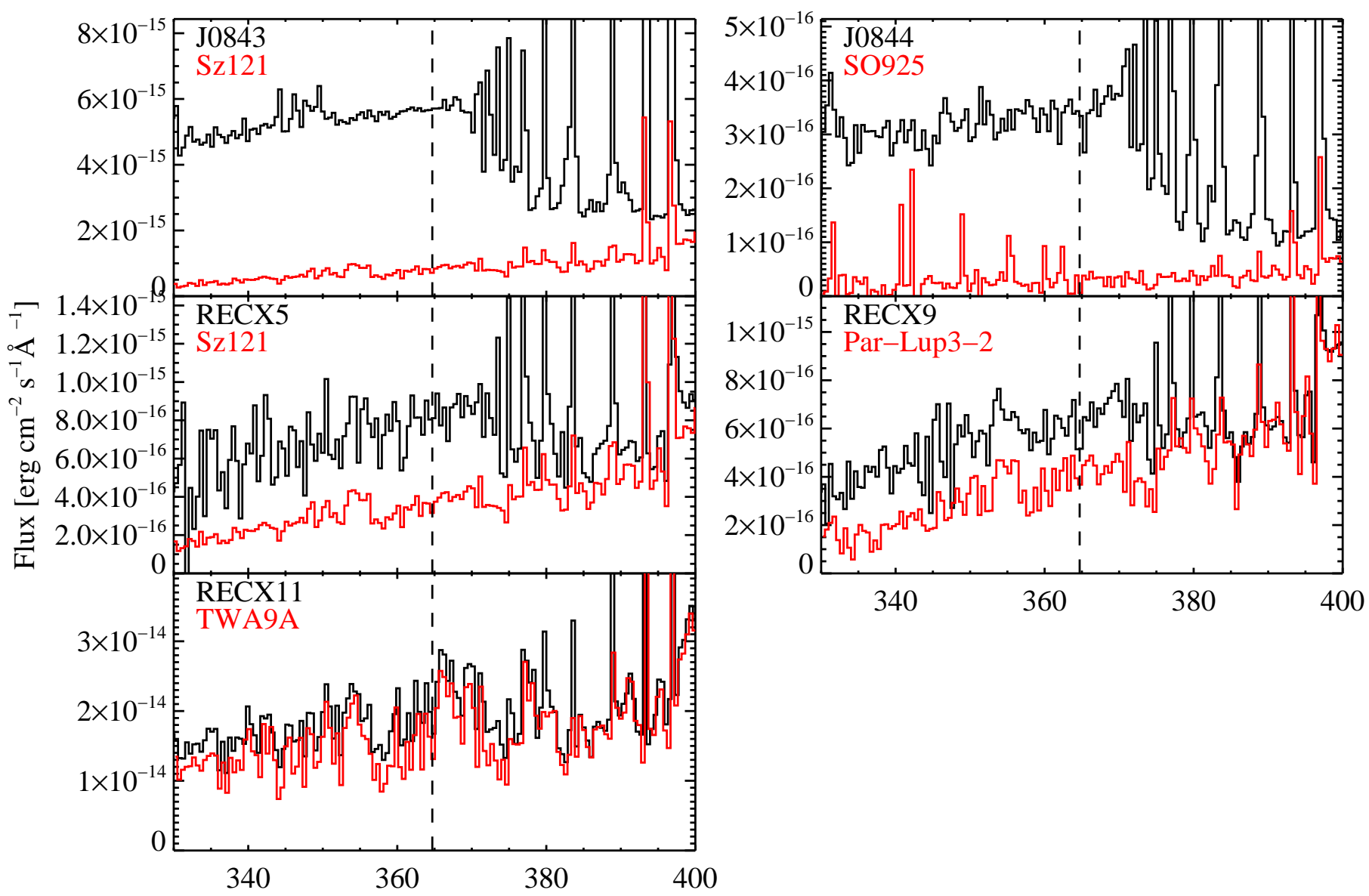

Fig. 7. As in Fig. 6. J0843, J0844, RECX 5 and RECX 9 show clear indications of UV-excess. For RECX 11, a slight excess is seen.

\subsection{Notes on individual objects}

RECX 12 and J0838 both show a slightly enhanced Balmer jump (Sect. 4.1), along with an equivalent width of the $\mathrm{H} \alpha$ line which is consistent with chromospheric emission. We therefore categorize both stars as non-accreting. However, the status of RECX 12 as a non-accretor is uncertain. The $\mathrm{H} \alpha$ line has weak line wings that are at levels of less than $10 \%$ of the peak flux and are asymmetric towards the blue side of the line. The $\mathrm{H} \alpha 10 \%$ width $\left(200 \pm 20 \mathrm{~km} \mathrm{~s}^{-1}\right)$ lies at the threshold for accretion (e.g., White \& Basri 2003; Jayawardhana et al. 2003; Natta et al. 2004). Three He I transitions are detected above $3 \sigma$ for RECX 12, but their equivalent widths are lower than any of the clearly accreting objects in the sample and are consistent with strong chromospheric emission. The upper limit on $L_{\text {acc }} / L_{*}$, as derived from continuum excess and $\mathrm{H} \alpha$ emission, is higher than for non-accreting objects of similar spectral types in the $\eta$ Cha cluster (see Fig. 8a,b). If the emission were interpreted as accretion, then $\dot{M}$ determined from the $\mathrm{H} \alpha$ transition and the continuum emission would agree well with each other, while the $\mathrm{H} \beta$ and $\mathrm{He}$ I emission lines would yield accretion rates $2-3$ times higher, and $\dot{M}$ would therefore be between $1.5-5.0 \times 10^{-10} M_{\odot} /$ yr. However, since some nonaccreting young stars share these characteristics (e.g., the PMS TWA15A in Manara et al. 2013b), we believe this borderline case is more likely to be a source with strong chromospheric emission.

The detection of [O I] emission in J0841 is unusual, since accretion is not detected in either $\mathrm{H} \alpha$ or in the Balmer continuum. However, a disk is detected from excess infrared emission (e.g., Sicilia-Aguilar et al. 2009). The He I $\lambda 402.6$ emission in this object is barely detected. Emission of the [O I] line can originate from disk winds, which can be related to the accretion process (e.g., Hartigan et al. 1995; Nisini et al. 2018). Mass accretion rates between $1.2-14 \times 10^{-11} M_{\odot} / \mathrm{yr}$ would be required to produce the luminosity of the [OI] line $\left(L_{\text {line }}\right)$, assuming a $L_{\text {acc }}-L_{\text {line }}$ relation from Natta et al. (2014). The upper limit on $\dot{M}$ from continuum excess emission of $\dot{M} \approx 0.6 \times 10^{-11} M_{\odot} / \mathrm{yr}$ falls slightly below this range. Hence, the origin of [O I] emission in J0841 is unclear.

The H $\alpha$ emission of RECX 7 shows a double-peaked profile and has been classified as a spectroscopic binary in the literature (Mamajek et al. 1999a; Lyo et al. 2003). The equivalent width of each component is in agreement with chromospheric emission. The peaks are separated by $\Delta v \approx 185 \mathrm{~km} \mathrm{~s}^{-1}$. An indication of $\mathrm{H} \beta$ emission may be seen in the low-velocity component (see lower right panel of Fig. 11), but is confused by absorption lines and is not considered significant.

\subsection{Mass accretion rates from hydrogen emission lines}

Table 6 lists $L_{\text {acc }}$ and $\dot{M}$ values derived from the line luminosities of $\mathrm{H} \alpha$ and $\mathrm{H} \beta$ using empirical $L_{\text {acc }}-L_{\text {line }}$ relations (Alcalá et al. 2014). The ratio $L_{\text {acc, } \mathrm{H} \alpha} / L_{*}$ is shown in Fig. 8b.

For J0843, the accretion rates derived from different tracers agree within $10 \%$. For J0844, RECX 5, and RECX 9, $\dot{M}_{\mathrm{H} \alpha}$ is lower by about a factor of two; however, $\dot{M}_{\mathrm{H} \beta}$ is closer to and within errors of $\dot{M}$ obtained from the continuum excess. 


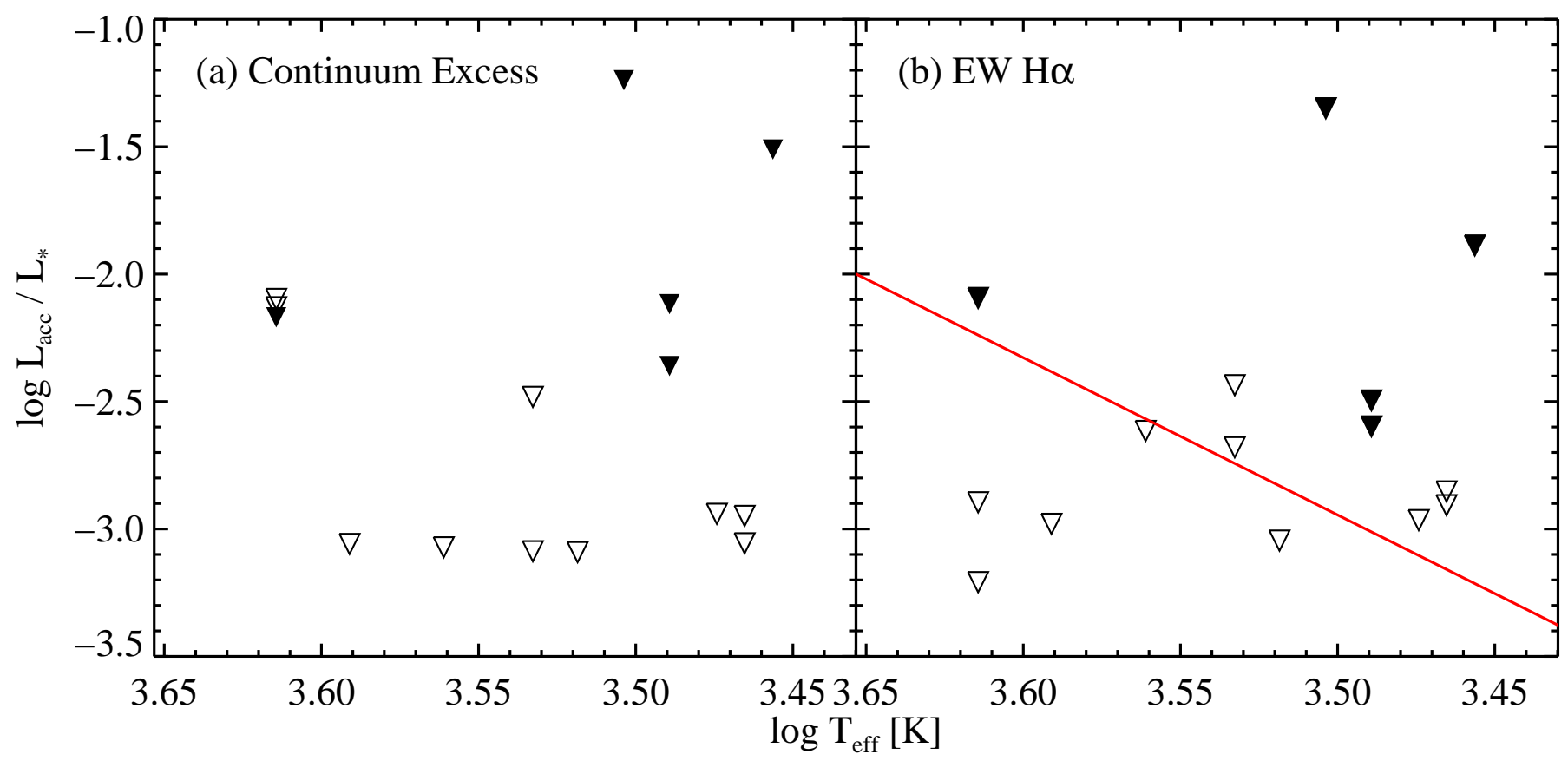

Fig. 8. Accretion luminosity (in units of $L_{*}$ ) vs. $T_{\text {eff }}$ for accreting PMS (filled triangles) and upper limits for non-accreting PMS (empty triangles). In panel $(a), L_{\mathrm{acc}}$ is derived from the continuum excess radiation and in panel $(b)$ from the $\mathrm{H} \alpha$ equivalent width. The red line in panel $(b)$ shows the chromospheric contribution from hydrogen emission lines to $L_{\text {acc }}$ (Manara et al. 2013b).

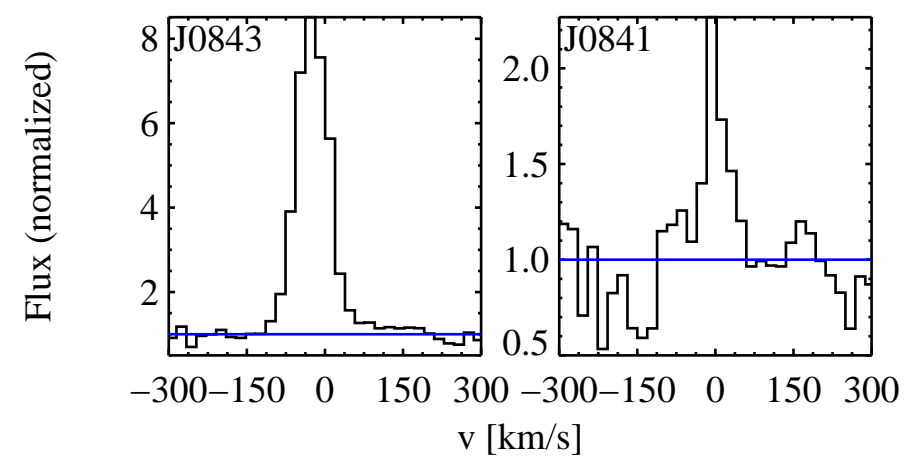

Fig. 9. Continuum normalized [O I] $\lambda 630.0 \mathrm{~nm}$ line profiles in J0843 and J0841. In J0843, the line is blueshifted. The continuum level is indicated by a blue line.

A possible reason for this discrepancy could be that the $\mathrm{H} \alpha$ line becomes easily optically thick and may be influenced by outflows (e.g., Alcalá et al. 2014). Accretion rates, which are derived from $L_{\text {line, } \mathrm{H} \alpha}$, agree closely with the accretion rates determined by direct modeling of the $\mathrm{H} \alpha$ line profile (Lawson et al. 2004).

The $\mathrm{H} \alpha$ equivalent width of RECX 11 is significantly higher than for the other K stars in this sample (RECX 1 and RECX 7) and falls in the range typically found for accreting stars at this spectral type (White \& Basri 2003), despite weak continuum emission (see Fig. 8). The $\mathrm{H} \alpha$ line of RECX 11 is also affected by redshifted absorption from the accretion flow (see also Ingleby et al. 2011). The values of $\dot{M}_{\mathrm{H} \alpha}$ and $\dot{M}_{\mathrm{H} \beta}$ are higher, but within the uncertainties of $\dot{M}$ determined from the UV-excess.

\section{Discussion}

\subsection{Detectability of mass accretion}

$\mathrm{H} \alpha$ emission is detected in all sources, generated by either accretion flow or chromospheric activity. As seen in Fig. 8b, the $L_{\text {acc }} / L_{*}$ ratio of accreting stars is higher than the upper limits inferred for non-accreting PMS. The upper limits on $L_{\mathrm{acc}, \mathrm{H} \alpha} / L_{*}$ are consistent with the threshold for "accretion noise" inferred by Manara et al. (2013b).

Detection limits for mass accretion rates from UV-excess depend on the spectral type of the star. For M-stars, the detection limits are at $\dot{M} \approx 1 \times 10^{-11} M_{\odot} /$ yr (Table 6). For the K-stars RECX 7 and RECX $1\left(\log T_{\text {eff }}>3.6\right)$, the upper limits of $\dot{M} \approx 4-8 \times 10^{-10} M_{\odot} /$ yr are higher than $\dot{M}$ of the accretor RECX 11; however, these upper limits do not reflect the detection limit, rather the large uncertainty in fitting the weak UV-excess emission in the warmer K-stars (see Sect. 4.3).

\subsection{Variability}

The accretion rate has been measured on four of the five accretors in $\eta$ Cha identified here. Our accretion rates obtained from excess Balmer continuum emission are consistent with past measurements using similar approaches, but in several cases the values are different from the $\mathrm{H} \alpha$-based measurements.

J0843: our accretion rate is similar to the UV-excess accretion rate $8 \times 10^{-10} M_{\odot} /$ yr measured by Ingleby et al. (2013) and is 1.25 times lower than the rate of $1 \times 10^{-9} M_{\odot} /$ yr measured from modeling the $\mathrm{H} \alpha$ line by Lawson et al. (2004). All three measurements agree within the errors.

RECX 9: our accretion rate is higher than previous measurements by a factor of three (Lawson et al. 2004; $4 \times 10^{-11} M_{\odot} / \mathrm{yr}$ ). The $\mathrm{H} \alpha$ line profile and equivalent width from Fig. 1 in Lawson et al. (2004) are similar to those measured in this work (central panel in Fig. 10), so the differences are likely the result of methodology.

RECX 5: as with RECX 9, the $\dot{M}$ of RECX 5 measured here is two times larger than that obtained from $\mathrm{H} \alpha$ modeling 

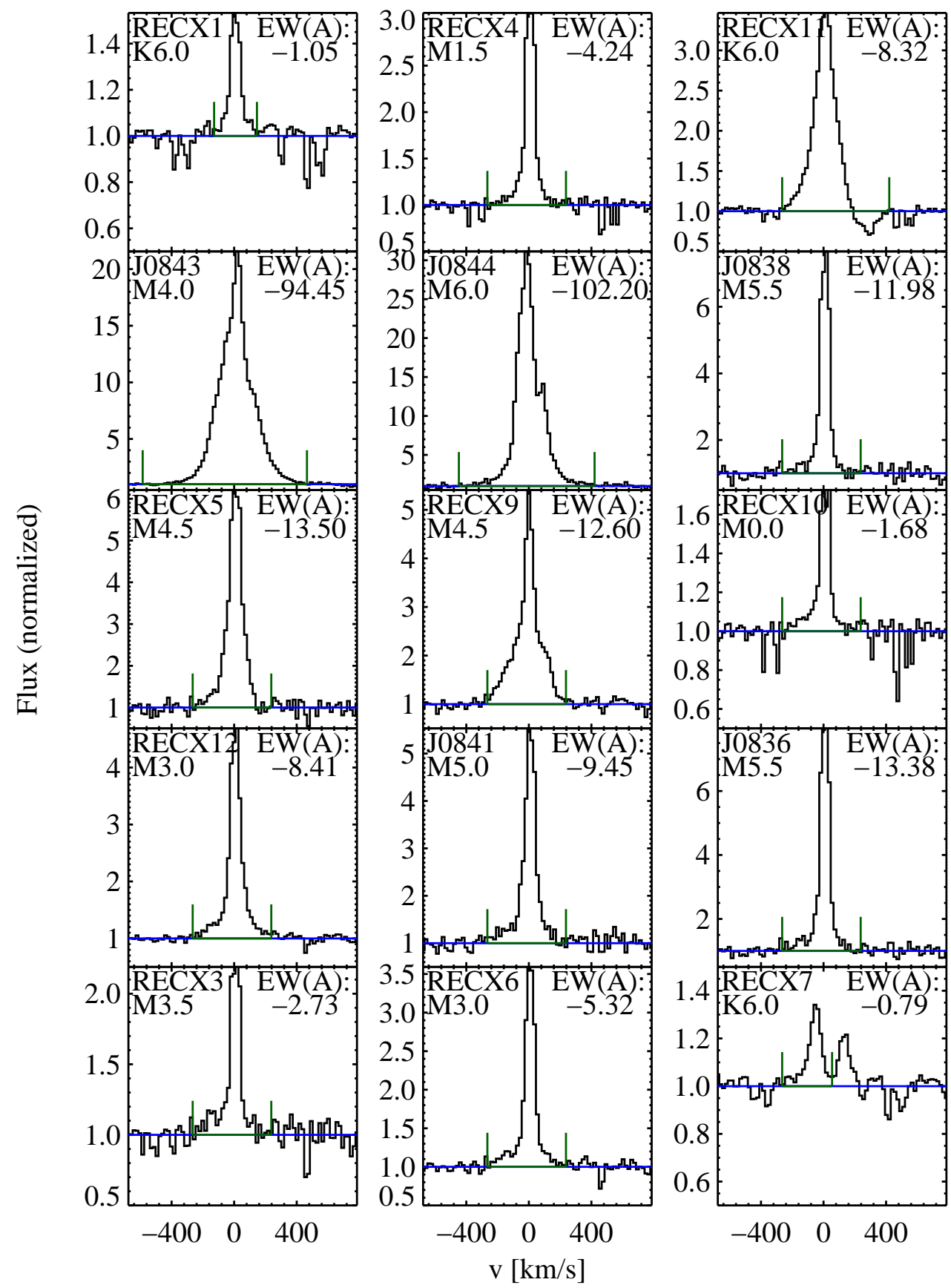

Fig. 10. Continuum normalized $\mathrm{H} \alpha$ line profiles in the $\eta$ Cha association. Green lines denote the integration boundaries for the determination of line flux and equivalent width. The continuum level is indicated by a blue line. Each panel shows the object's name, its spectral type, and the measured equivalent width (EW).

(5 $\times 10^{-11} M_{\odot} / \mathrm{yr}$; Lawson et al. 2004). This difference is unlikely due to variability because the Lawson et al. spectrum had a much stronger and broader $\mathrm{H} \alpha$ emission: an equivalent width of $-35 \AA$, a $10 \%$ width of $330 \mathrm{~km} \mathrm{~s}^{-1}$, and a FWHM of $160 \mathrm{~km} \mathrm{~s}^{-1}$ in the Lawson et al. spectrum, compared to $-14 \AA, 220 \mathrm{~km} \mathrm{~s}^{-1}$, and $105 \mathrm{~km} \mathrm{~s}^{-1}$ in our spectrum. The variability in $\mathrm{H} \alpha$ emission from RECX 5 had been found previously (Jayawardhana et al. 2006; Murphy et al. 2011).

RECX 11: the value of $\dot{M}$ from this work is similar to the value found by previous measurements of UV-excess $(1.7 \times$ $10^{-10} M_{\odot} / y r$; Ingleby et al. 2011, 2013). However, both of these accretion rates are five times higher than that determined from
$\mathrm{H} \alpha$ line profile modeling $\left(4 \times 10^{-11} M_{\odot} / \mathrm{yr}\right.$; Lawson et al. 2004). The $\mathrm{H} \alpha$ line is stronger in our observations $(-8.3 \AA$ equivalent width) than in the Lawson et al. spectrum ( $-3 \AA$ ), so the different accretion rates may result from methodology, variability, or uncertainty in accretion measured from the weak UV excess.

J0844: no previous direct measurement of mass accretion onto J0844 exists in the literature. The equivalent width of the $\mathrm{H} \alpha$ line in the X-shooter spectrum is nearly twice as high as previously reported (-57.8 $\AA$; Song et al. 2004), with a range in variability consistent with that seen from other sources (Costigan et al. 2012). The $\dot{M}$ of J0844 is located at the high end of the scatter in the $\dot{M}$ of accreting low-mass stars (Fig. 12; see Sect. 6.3). 

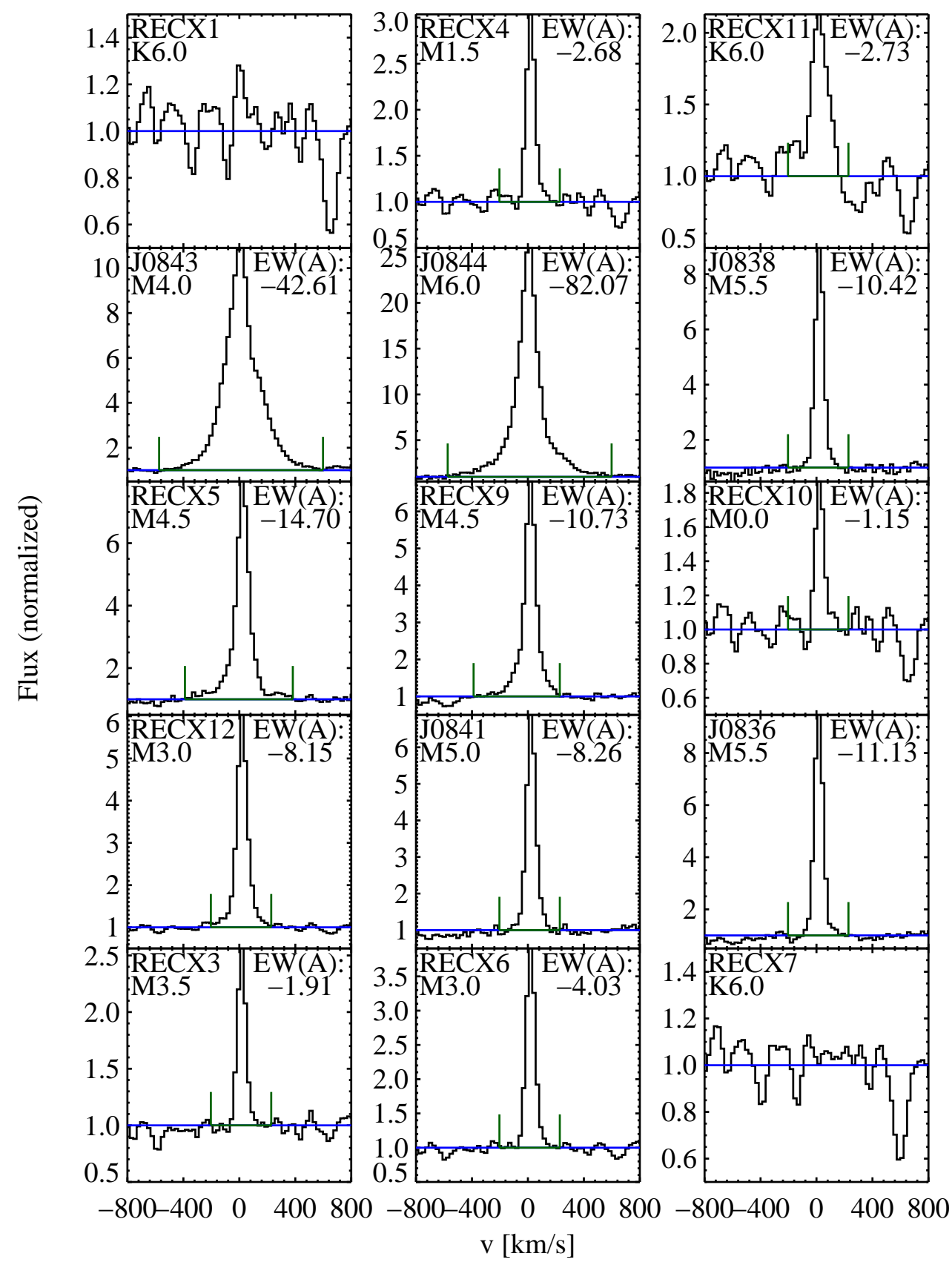

Fig. 11. Line profiles of the $\mathrm{H} \beta \lambda 486.13 \mathrm{~nm}$ transition. Symbols and colors as in Fig. 10.

To summarize, for J0843 and RECX 11 the accretion rates computed here are similar to previous accretion rates based on UV-excess. This similarity indicates that the methodology is stable and that these two objects had accretion rates that were consistent on time baselines of a few weeks, the time between the Ingleby et al. (2013) spectra and those obtained here.

On the other hand, the accretion rates for RECX 9 and RECX 5 measured from the UV-excess are discrepant with those measured from $\mathrm{H} \alpha$. If real and not the result of different methodologies, then these changes are consistent with the accretion variability of 0.37 dex inferred from $\mathrm{H} \alpha$ monitoring (Costigan et al. 2012), which may be correlated with stellar rotation as the accretion flow is seen from different sides at different phases in the period. This variability may instead indicate quasi-periodic bursts (e.g., Cody et al. 2017). Changes in the accretion rate and line equivalent widths and profiles are not necessarily correlated because the excess accretion continuum emission is thought to be produced by the accretion shock, while the $\mathrm{H}$ line emission is likely produced in the accretion funnel flows (see modeling of line profiles by Alencar et al. 2012).

\subsection{Comparisons to other low-mass star forming regions}

Figures 12 and 13 compare the UV-excess measurements of $L_{\text {acc }}$ and $\dot{M}$ of $\eta$ Cha members with those measured in stars in the Lupus and Chamaeleon I star forming regions (Alcalá et al. 2014, 2017; Manara et al. 2016). The locus of $\eta$ Cha objects is consistent with accretors in other regions. Therefore, the accretion properties appear to be similar.

To support this conclusion, we compare the mass accretion rates of the $\eta$ Cha association to those of other clusters 


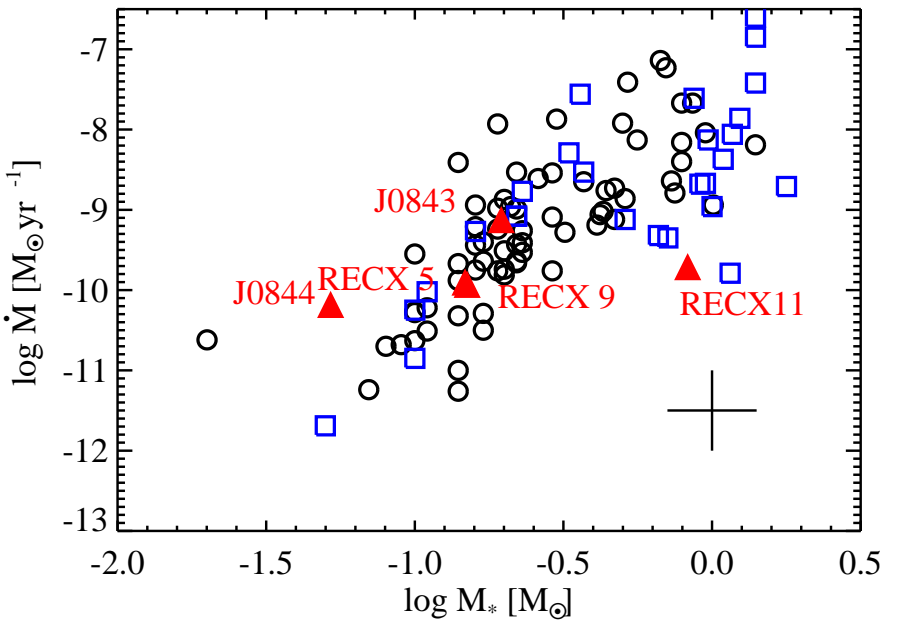

Fig. 12. Mass accretion rate vs. stellar mass for objects in the $\eta$ Cha cluster (red filled triangles; this work), the Lupus (black empty circles; Alcalá et al. 2017), and Chamaeleon I star forming regions (blue empty squares; Manara et al. 2016). RECX 5 and RECX 9 overlap in this presentation as they differ only slightly in stellar mass and mass accretion rate. Typical errors are indicated in the lower right corner.

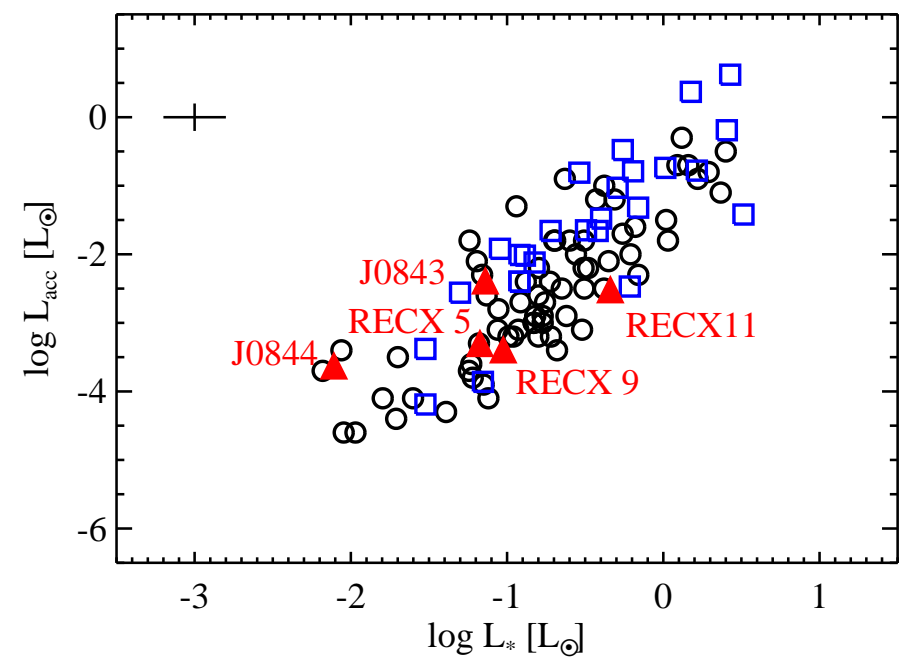

Fig. 13. Accretion luminosity vs. stellar mass for objects in the $\eta$ Cha cluster (red filled triangles; this work), the Lupus (black empty circles; Alcalá et al. 2017), and Chamaeleon I star forming regions (blue empty squares; Manara et al. 2016). Typical errors are indicated in the upper left corner.

with different ages. The measurements have been compiled from the literature for the $\rho$ Ophiucus (Manara et al. 2015), Lupus (Alcalá et al. 2017), Chamaeleon I (Manara et al. 2016), and $\sigma$ Orionis (Rigliaco et al. 2011) star forming regions. These clusters have been selected because the mass accretion rates were determined with a similar technique (except for $\rho$ Ophiucus, for which $\dot{M}$ was determined from emission lines; Manara et al. 2015) and the observations were conducted with the same instrument (X-shooter). We adopt cluster ages of $0.5 \mathrm{Myr}$ for $\rho$ Ophiucus (Greene \& Meyer 1995; Luhman \& Rieke 1999; Mohanty et al. 2005), 2 Myr for Chamaeleon I, 2.5 Myr for $\sigma$ Orionis (both Fang et al. 2013, and references therein), 3 Myr for Lupus (Alcalá et al. 2014, 2017), and $5 \mathrm{Myr}$ for the $\eta$ Cha Association. The accretion rate for each star is normalized by the relationship $\dot{M} \propto M_{*}^{2.0}$ (based on previous estimates with an exponent $\sim 2.0$ from, e.g., Hartmann et al. 2016; Manara et al. 2017).

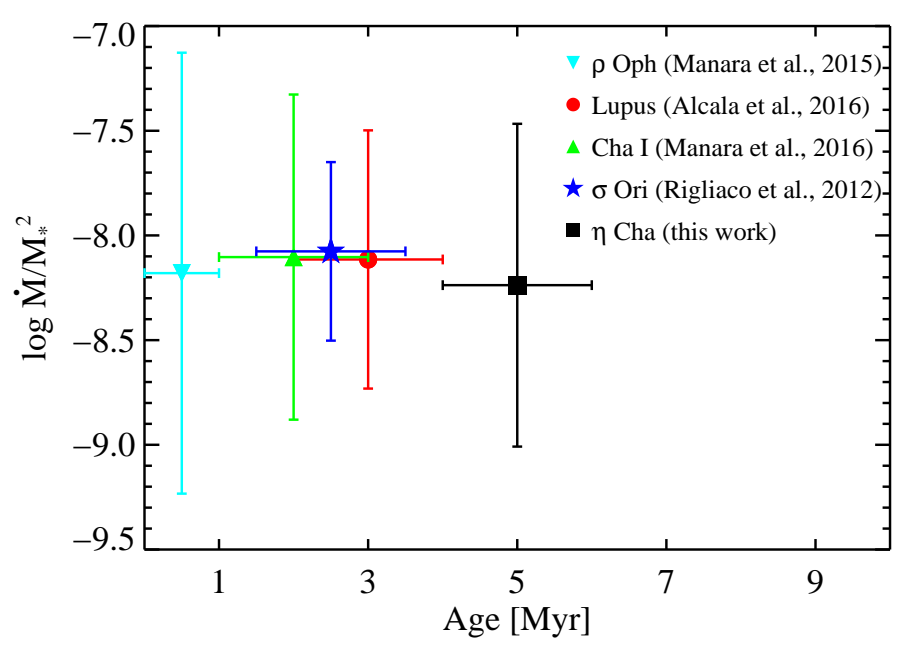

Fig. 14. Mass accretion rates normalized by stellar mass squared at cluster ages of individual star forming regions. The mass accretion rate $\dot{M}$ of each star has been divided by $M_{*}^{2}$ and combined by the median for each cluster. $\dot{M}$ is given in units of $M_{\odot} / \mathrm{yr}$ and the stellar mass $M_{*}$ is given in units of $M_{\odot}$. Stars in the $\eta$ Cha association are shown as filled black square. The other clusters are shown as a cyan inverted triangle for $\rho$ Ophiucus, as a red circle for Lupus, as a green triangle for Chamaeleon I, and as a blue star for $\sigma$ Orionis. The error bars indicate the standard deviation in logarithmic scale of the normalized mass accretion rates.

The median mass accretion rates agree well for all clusters, despite differences in age (Fig. 14). Disk dispersal is expected to be governed by viscous accretion at least in some phases of its evolution, which implies a decrease in mass accretion rate with time (e.g., Hartmann et al. 1998; Alexander et al. 2014). Among stars with ongoing accretion, the decrease in accretion rate with time is not detected.

However, drawing conclusions on the physical description of disk accretion from these comparisons is challenging for several reasons (see also Manara et al. 2016; Hartmann et al. 2016). First, while the average accretion rate may not change, the fraction of stars with disks and with ongoing accretion decreases with time (e.g., Haisch et al. 2001; Hernández et al. 2008; Fedele et al. 2010). Second, stars in a single cluster may have an age spread, in which case the stars with disks may be younger than the average cluster age. Finally, according to models of viscous accretion, the change in accretion rate with stellar age will flatten out, i.e., it will decrease with stellar age (e.g., Hartmann et al. 1998). The $\eta$ Chamaeleontis cluster may therefore be biased towards higher initial disk masses as these live longer than their lower mass counterparts. Comparisons of older clusters to younger ones are therefore difficult (Sicilia-Aguilar et al. 2010). This effect may be enhanced by the possible onset of photo-evaporation, which could quickly remove disks once the mass accretion rate drops below about $10^{-10} M_{\odot} / \mathrm{yr}$ for solar-mass stars (Clarke et al. 2001; Gorti et al. 2009).

We therefore investigate how the accretion rates of stars of similar mass compare among clusters of different ages, and how they relate to models of viscous evolution (Fig. 15). As the $\eta$ Cha association hosts only five accreting objects that span an order of magnitude in stellar mass, the cluster samples are divided into three mass bins and each $\eta$ Cha object is drawn separately. The mass ranges have been chosen around the mass of J0844 $\left(0.025<M_{\odot}<0.1\right.$; left panel); of J0843, RECX 5, and RECX 9 (0.1 $<M_{\odot}<0.3$; middle panel); and of RECX 11 $\left(0.65<M_{\odot}<0.95\right.$; right panel). For the clusters, the mean in 


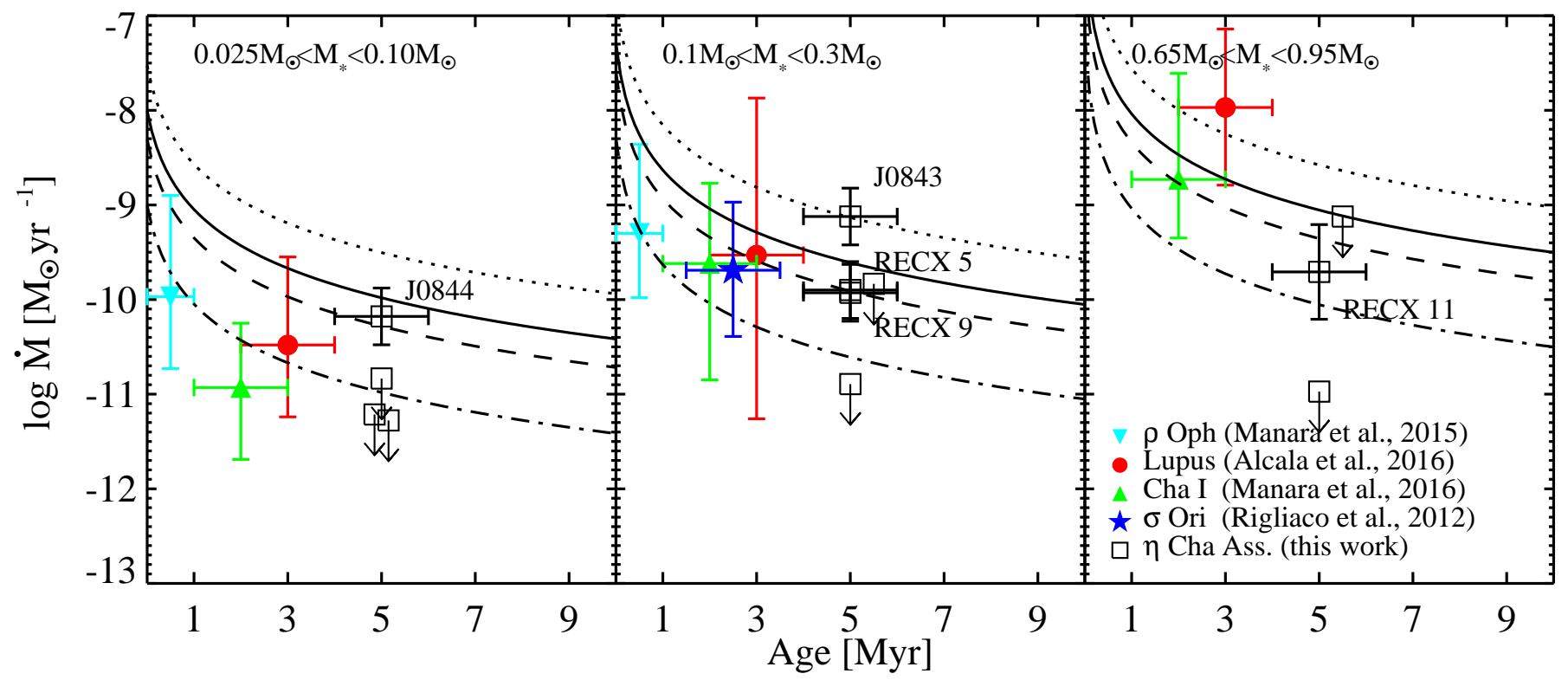

Fig. 15. Mass accretion rate vs. cluster age for three different stellar mass ranges. The ranges are indicated in each panel. Stars in the $\eta$ Cha association are shown as empty black squares. Upper limits are drawn as arrows for objects with no detected accretion, otherwise colors and symbols as in Fig. 14. The dotted, solid, dashed and dash-dotted lines indicate fiducial models for viscous accretion with disk masses of 30\%, 10\%, 5\%, and $1 \%$ of the mass of the central star, respectively. The stellar mass used in the model is $M_{*}=0.05 M_{\odot}$ in the left, $M_{*}=0.15 M_{\odot}$ in the middle, and $M_{*}=0.8 M_{\odot}$ in the right panel (see details and references in text).

logarithmic scale of $\dot{M}$ is given. It is only shown if a single mass bin contained at least three objects. The error bars of $\dot{M}$ are the minimum and maximum accretion rate in each mass bin. While upper limits on $\dot{M}$ are drawn as arrows for non-accreting objects in the $\eta$ Cha association, upper limits have not been included for other clusters.

Fiducial models of accretion by viscous evolution (Hartmann et al. 1998) are for stellar masses of $0.05,0.15$, and $0.8 M_{\odot}$, with initial disk masses of $30 \%, 10 \%, 5 \%$, and $1 \%$ times the mass of the central star. This simple parametric model assumes $\alpha=10^{-2}$, $R_{1}=10 \mathrm{AU}$, and $T_{100 \mathrm{AU}}=10 \mathrm{~K}$ (see Hartmann et al. 1998 for more details on the parameters).

In the lowest mass bin, the time evolution of accretion between $\rho$ Ophiucus and Lupus and Chamaeleon I is nicely traced by a viscous accretion model with $M_{*}=0.05 M_{\odot}$ and a disk mass of $M_{\text {disk }}=0.0005 M_{\odot}$ (dash-dotted model in the left panel of Fig. 15). At the age of the $\eta$ Cha cluster, mass accretion rates expected from this model would be below the detection limit of the method presented here, as indicated by the upper limits of non-accreting objects. On the other hand, the $\dot{M}$ of J0844 lies at the high end of the range of mass accretion rates found in Lupus and Chamaeleon I, which could be explained if J0844 had a high initial disk mass. J0843 also shows an enhanced accretion rate.

RECX 5 and RECX 9 are accreting at rates that are lower but within the range of accretion rates in Lupus, Chamaeleon I, and $\sigma$ Orionis. This difference is in agreement with viscous accretion models. RECX 11 shows significantly lower $\dot{M}$ than that seen in the other clusters for stars of similar mass. In direct comparison to Chamaeleon I, this difference is in agreement with expectations from simple models of viscous disk accretion (see Fig. 15).

\section{Conclusions}

We present a revised analysis of the mass accretion and stellar properties of 15 low-mass stars in the nearby $\eta$ Cha association. Thanks to the simultaneous, broad wavelength range coverage, and flux-calibration accuracy of VLT/X-shooter we determined the spectral type, extinction, and accretion luminosity in a self-consistent way. Of the 15 low-mass stars studied here, we detected ongoing mass accretion in 5 systems. Once compared with literature values, we find that the mass accretion rates are consistent within errors with previous studies for which UV excess measurements exist, and deviating for most objects, when compared to results from $\mathrm{H} \alpha$ modeling due to methodological differences. We also report a mass accretion rate for $\mathrm{J} 0844$, for which no direct measurement of mass accretion has been reported in the literature. The derived mass accretion rates in the $\eta$ Cha cluster are similar to the values measured in younger star forming regions.

Acknowledgements. We kindly thank the anonymous referee for the careful reading, the detailed suggestions, and thorough comments that helped to significantly improve the readability and to strengthen the scientific message of the paper. We thank C.F. Manara for kindly providing us with the data of non-accreting PMS and for the discussion. We also thank Paula Teixeira for help with the observations, and together with Kevin Covey and Adam Kraus for help in preparing the proposal. M.R. is a fellow of the International Max Planck Research School for Astronomy and Cosmic Physics (IMPRS) at the University of Heidelberg. D.F. acknowledges support from the Italian Ministry of Education, Universities and Research project SIR (RBSI14ZRHR). G.J.H. is supported by general grant 11473005 awarded by the National Science Foundation of China. This research has made use of the VizieR catalogue access tool, CDS, Strasbourg, France.

\section{References}

Alcalá, J. M., Natta, A., Manara, C. F., et al. 2014, A\&A, 561, A2

Alcalá, J. M., Manara, C. F., Natta, A., et al. 2017, A\&A, 600, A20

Alencar, S. H. P., Bouvier, J., Walter, F. M., et al. 2012, A\&A, 541, A116

Alexander, R., Pascucci, I., Andrews, S., Armitage, P., \& Cieza, L. 2014, Protostars and Planets VI, 475

Allard, F., Homeier, D., \& Freytag, B. 2012, Royal Society of London Philosophical Transactions Series A, 370, 2765

Baraffe, I., Homeier, D., Allard, F., \& Chabrier, G. 2015, A\&A, 577, A42

Basri, G., \& Batalha, C. 1990, ApJ, 363, 654

Basri, G., \& Bertout, C. 1989, ApJ, 341, 340

Bell, C. P. M., Mamajek, E. E., \& Naylor, T. 2015, MNRAS, 454, 593

Bertout, C., Basri, G., \& Bouvier, J. 1988, ApJ, 330, 350 
Calvet, N., \& Gullbring, E. 1998, ApJ, 509, 802

Cardelli, J. A., Clayton, G. C., \& Mathis, J. S. 1989, ApJ, 345, 245

Clarke, C. J., Gendrin, A., \& Sotomayor, M. 2001, MNRAS, 328, 485

Cody, A. M., Hillenbrand, L. A., David, T. J., et al. 2017, ApJ, 836, 41

Costigan, G., Scholz, A., Stelzer, B., et al. 2012, MNRAS, 427, 1344

Cutri, R. M., et al. 2012, VizieR Online Data Catalog, 2311

Fang, M., van Boekel, R., Bouwman, J., et al. 2013, A\&A, 549, A15

Fedele, D., van den Ancker, M. E., Petr-Gotzens, M. G., \& Rafanelli, P. 2007, A\&A, 472, 207

Fedele, D., van den Ancker, M. E., Henning, T., Jayawardhana, R., \& Oliveira, J. M. 2010, A\&A, 510, A72

Gahm, G. F., Walter, F. M., Stempels, H. C., Petrov, P. P., \& Herczeg, G. J. 2008 A\&A, 482, L35

Gaia Collaboration (Brown, A. G. A., et al.) 2016, A\&A, 595, A2

Gorti, U., Dullemond, C. P., \& Hollenbach, D. 2009, ApJ, 705, 1237

Greene, T. P., \& Meyer, M. R. 1995, ApJ, 450, 233

Gullbring, E., Hartmann, L., Briceno, C., \& Calvet, N. 1998, ApJ, 492, 323

Gully-Santiago, M. A., Herczeg, G. J., Czekala, I., et al. 2017, ApJ, 836, 200

Haisch, Jr., K. E., Lada, E. A., \& Lada, C. J. 2001, ApJ, 553, L153

Hamann, F., \& Persson, S. E. 1992, ApJS, 82, 247

Hamuy, M., Suntzeff, N. B., Heathcote, S. R., et al. 1994, PASP, 106, 566

Hartigan, P., Edwards, S., \& Ghandour, L. 1995, ApJ, 452, 736

Hartmann, L., Calvet, N., Gullbring, E., \& D’Alessio, P. 1998, ApJ, 495, 385

Hartmann, L., Herczeg, G., \& Calvet, N. 2016, ARA\&A, 54, 135

Herczeg, G. J., \& Hillenbrand, L. A. 2008, ApJ, 681, 594

Herczeg, G. J., \& Hillenbrand, L. A. 2014, ApJ, 786, 97

Herczeg, G. J., \& Hillenbrand, L. A. 2015, ApJ, 808, 23

Herczeg, G. J., Cruz, K. L., \& Hillenbrand, L. A. 2009, ApJ, 696, 1589

Hernández, J., Hartmann, L., Calvet, N., et al. 2008, ApJ, 686, 1195

Hessman, F. V., Eisloeffel, J., Mundt, R., et al. 1991, ApJ, 370, 384

Ingleby, L., Calvet, N., Bergin, E., et al. 2011, ApJ, 743, 105

Ingleby, L., Calvet, N., Herczeg, G., et al. 2013, ApJ, 767, 112

Jayawardhana, R., Mohanty, S., \& Basri, G. 2003, ApJ, 592, 282

Jayawardhana, R., Coffey, J., Scholz, A., Brandeker, A., \& van Kerkwijk, M. H 2006, ApJ, 648, 1206

Jeffries, R. D., Oliveira, J. M., Naylor, T., Mayne, N. J., \& Littlefair, S. P. 2007 MNRAS, 376, 580

Lawson, W., \& Feigelson, E. D. 2001, in From Darkness to Light: Origin and Evolution of Young Stellar Clusters, eds. T. Montmerle, \& P. André, ASP Conf. Ser., 243, 591

Lawson, W. A., Crause, L. A., Mamajek, E. E., \& Feigelson, E. D. 2001, MNRAS, 321, 57
Lawson, W. A., Lyo, A.-R., \& Muzerolle, J. 2004, MNRAS, 351, L39 Lopez Martí, B., Jimenez Esteban, F., Bayo, A., et al. 2013, A\&A, 551, A46 Luhman, K. L., \& Rieke, G. H. 1999, ApJ, 525, 440 Luhman, K. L., \& Steeghs, D. 2004, ApJ, 609, 917

Lyo, A.-R., Lawson, W. A., Mamajek, E. E., et al. 2003, MNRAS, 338, 616 Lyo, A.-R., Lawson, W. A., \& Bessell, M. S. 2004, MNRAS, 355, 363 Mamajek, E. E., Lawson, W. A., \& Feigelson, E. D. 1999a, PASA, 16, 257

Mamajek, E. E., Lawson, W. A., \& Feigelson, E. D. 1999b, ApJ, 516, L77

Manara, C. F., Beccari, G., Da Rio, N., et al. 2013a, A\&A, 558, A114

Manara, C. F., Testi, L., Rigliaco, E., et al. 2013b, A\&A, 551, A107

Manara, C. F., Testi, L., Natta, A., \& Alcalá, J. M. 2015, A\&A, 579, A66

Manara, C. F., Fedele, D., Herczeg, G. J., \& Teixeira, P. S. 2016, A\&A, 585, A136

Manara, C. F., Testi, L., Herczeg, G. J., et al. 2017, A\&A, 604, A127

Megeath, S. T., Hartmann, L., Luhman, K. L., \& Fazio, G. G. 2005, ApJ, 634 L113

Modigliani, A., Goldoni, P., Royer, F., et al. 2010, in SPIE Conf. Ser., 7737, 28

Mohanty, S., Jayawardhana, R., \& Basri, G. 2005, ApJ, 626, 498

Murphy, S. J., Lawson, W. A., \& Bessell, M. S. 2010, MNRAS, 406, L50

Murphy, S. J., Lawson, W. A., Bessell, M. S., \& Bayliss, D. D. R. 2011, MNRAS, 411, L51

Natta, A., Testi, L., Muzerolle, J., et al. 2004, A\&A, 424, 603

Natta, A., Testi, L., Alcalá, J. M., et al. 2014, A\&A, 569, A5

Nisini, B., Antoniucci, S., Alcalá, J. M., et al. 2018, A\&A, in press, DOI: $10.1051 / 0004-6361 / 201730834$

Pecaut, M. J., \& Mamajek, E. E. 2013, ApJS, 208, 9

Rigliaco, E., Natta, A., Randich, S., et al. 2011, A\&A, 526, L6

Sicilia-Aguilar, A., Bouwman, J., Juhász, A., et al. 2009, ApJ, 701, 1188

Sicilia-Aguilar, A., Henning, T., \& Hartmann, L. W. 2010, ApJ, 710, 597

Simon, M., Schlieder, J. E., Constantin, A.-M., \& Silverstein, M. 2012, ApJ, 751, 114

Simon, M. N., Pascucci, I., Edwards, S., et al. 2016, ApJ, 831, 169

Song, I., Zuckerman, B., \& Bessell, M. S. 2004, ApJ, 600, 1016

Stelzer, B., Frasca, A., Alcalá, J. M., et al. 2013, A\&A, 558, A141

Valenti, J. A., Basri, G., \& Johns, C. M. 1993, AJ, 106, 2024

van den Ancker, M. E., Blondel, P. F. C., Tjin A Djie, H. R. E., et al. 2004, MNRAS, 349, 1516

van Leeuwen, F. 2007, A\&A, 474, 653

Vernet, J., Kerber, F., Mainieri, V., et al. 2009, in Highlights of Astronomy, Proceedings of the International Astronomical Union, 5, 535

Vernet, J., Dekker, H., D’Odorico, S., et al. 2011, A\&A, 536, A105

White, R. J., \& Basri, G. 2003, ApJ, 582, 1109 


\section{Appendix A: Spectra and best-fit models of accretors in the $\eta$ Cha cluster}

In Figs. A.1-A.5, the accreting PMSs in the $\eta$ Cha cluster are shown together with the models used to determine the accretion flux.

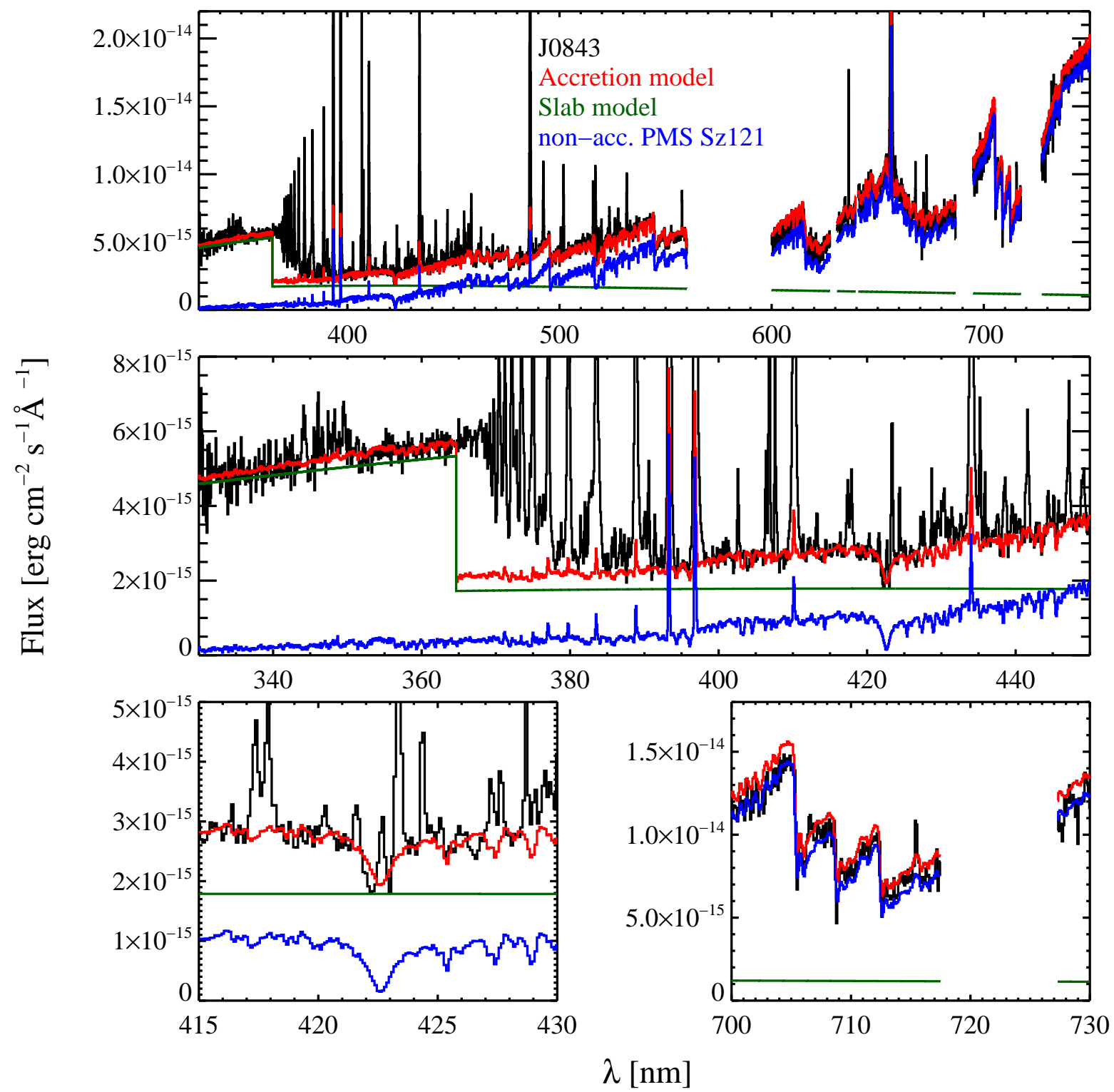

Fig. A.1. Accretion model (red) of PMS J0843 (black), using as a basis the non-accreting PMS Sz121 (blue; Manara et al. 2013b) and a plane parallel hydrogen slab (green; Valenti et al. 1993). The spectra have been re-binned to $0.1 \mathrm{~nm}$ resolution. 
M. Rugel et al.: X-shooter observations of low-mass stars in the $\eta$ Chamaeleontis association

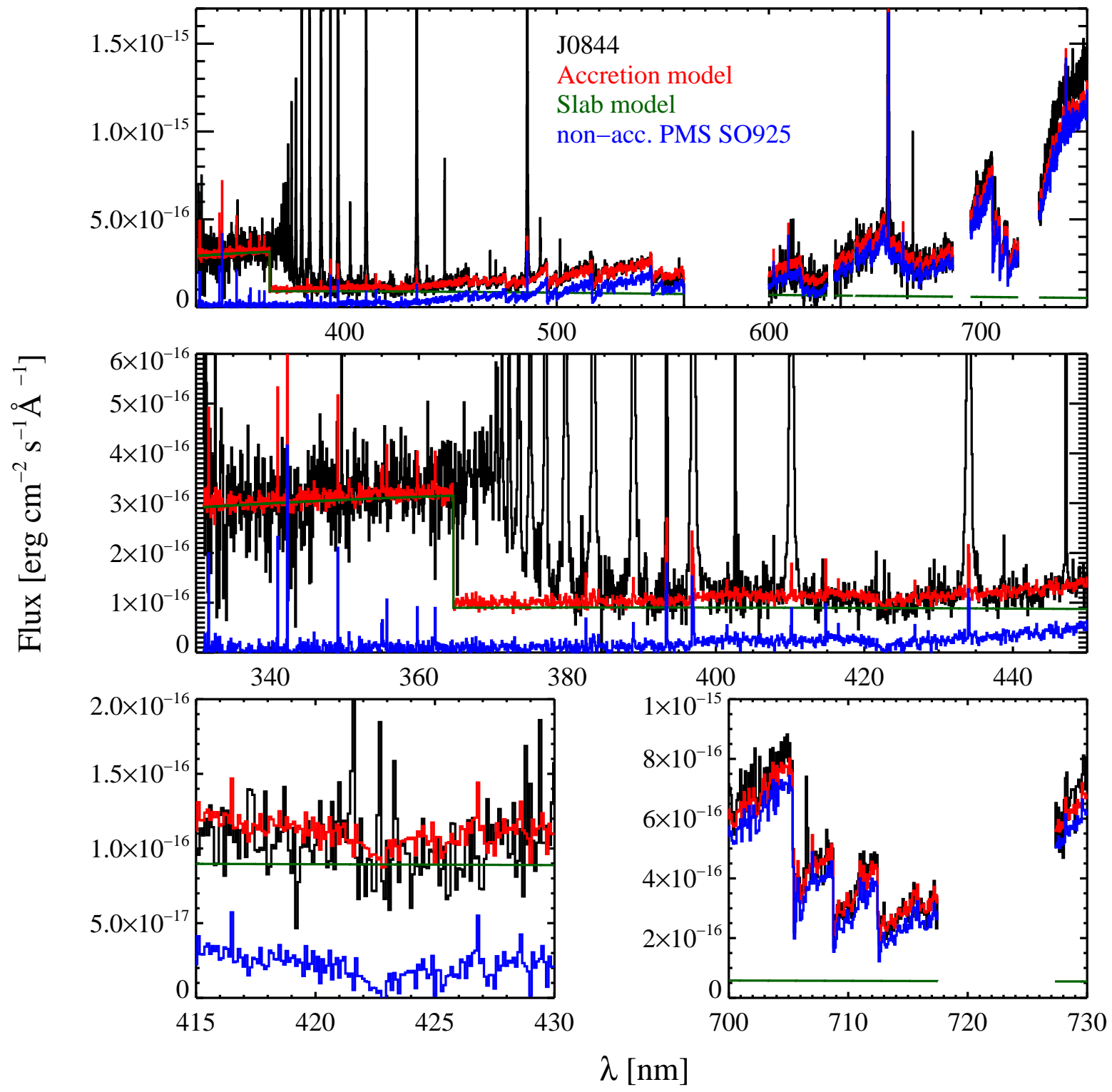

Fig. A.2. Accretion model of PMS J0844, using as a basis the non-accreting PMS SO925 (Manara et al. 2013b). Colors as in Fig. A.1. 


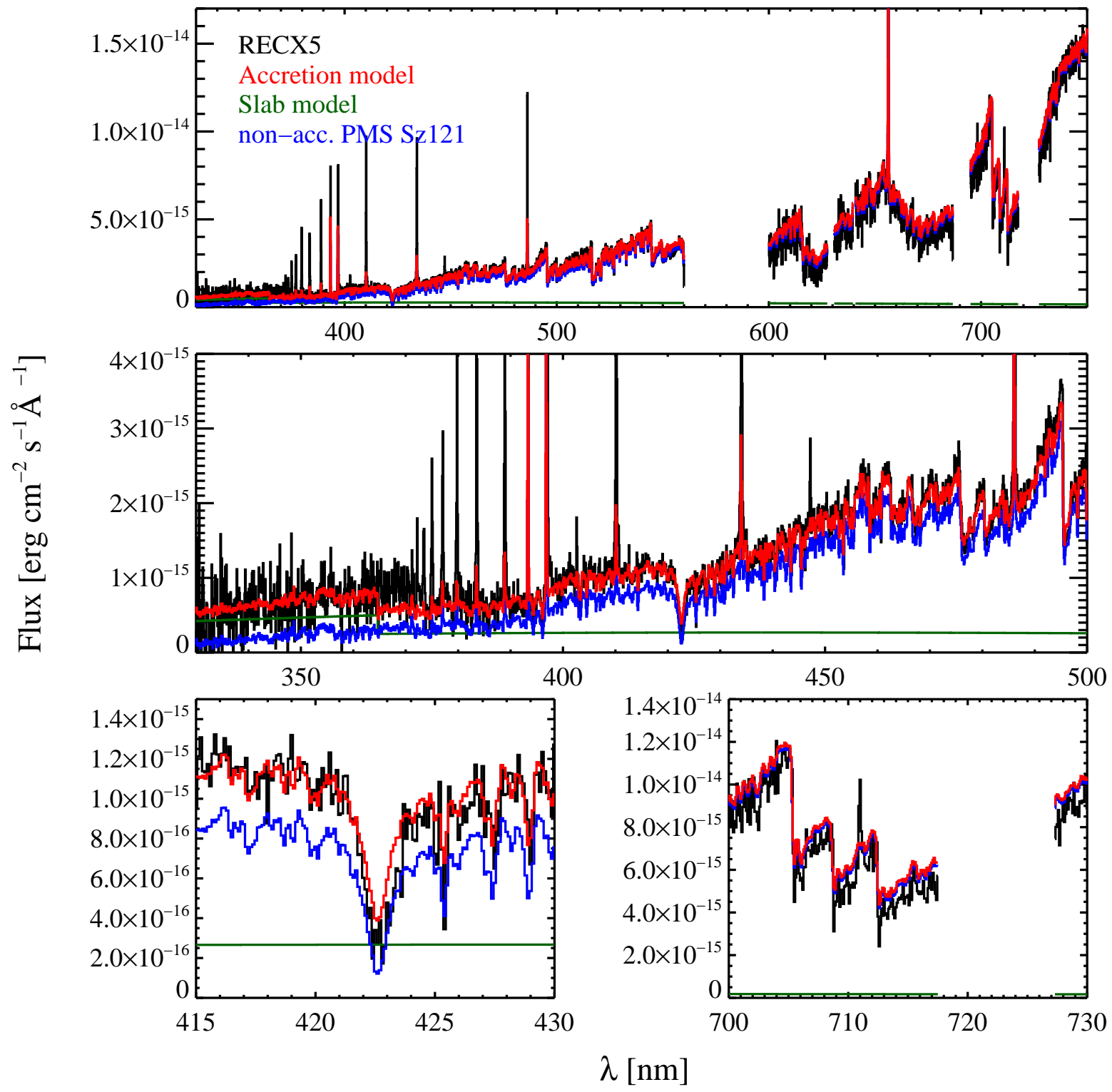

Fig. A.3. Accretion model to PMS RECX5, using as a basis the non-accreting PMS Sz121 (Manara et al. 2013b). Colors as in Fig. A.1. 
M. Rugel et al.: X-shooter observations of low-mass stars in the $\eta$ Chamaeleontis association

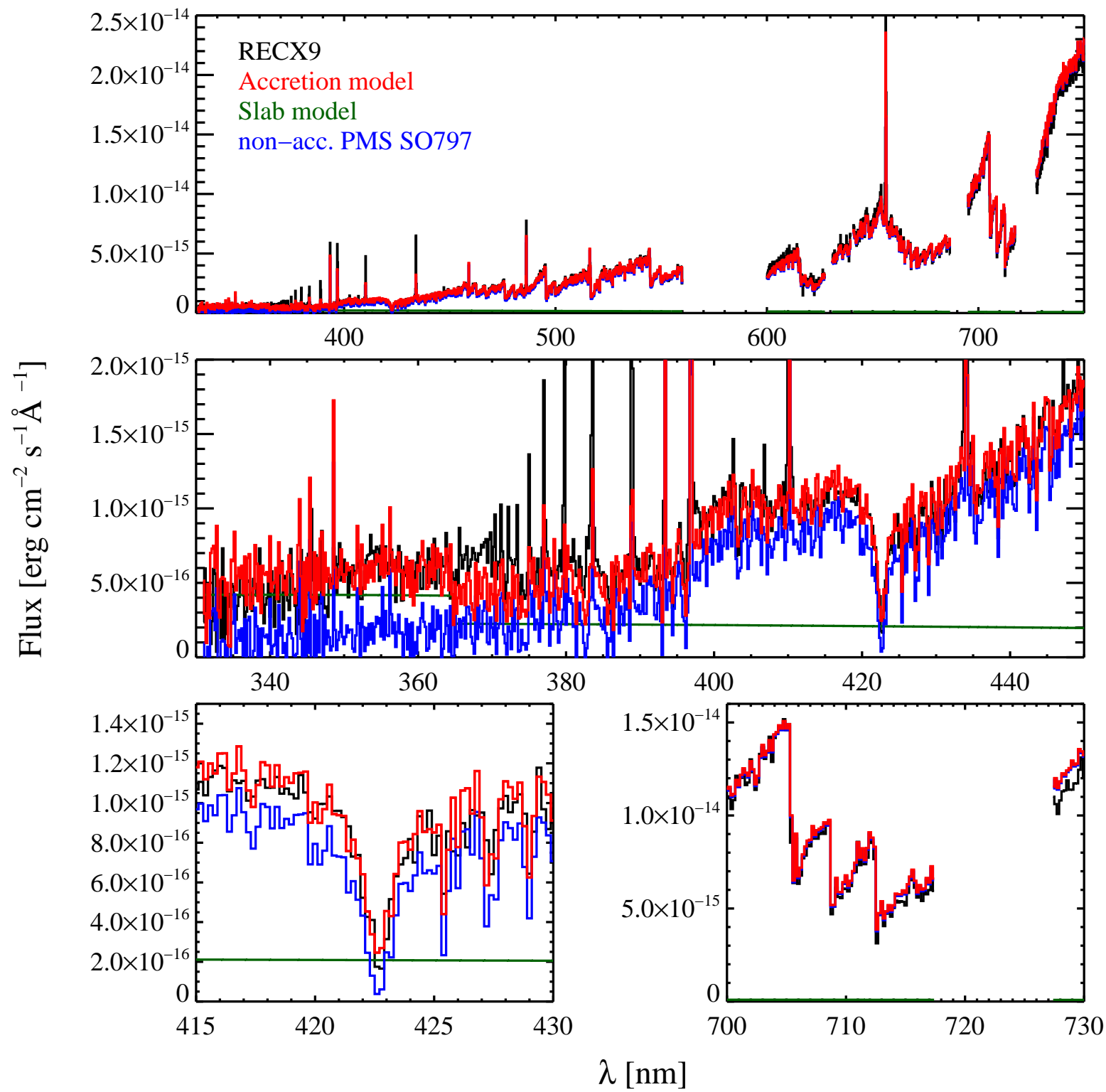

Fig. A.4. Accretion model to PMS RECX 9, using as a basis the non-accreting PMS SO797 (Manara et al. 2013b). Colors as in Fig. A.1. The spectra have been re-binned to $0.2 \mathrm{~nm}$ resolution. 

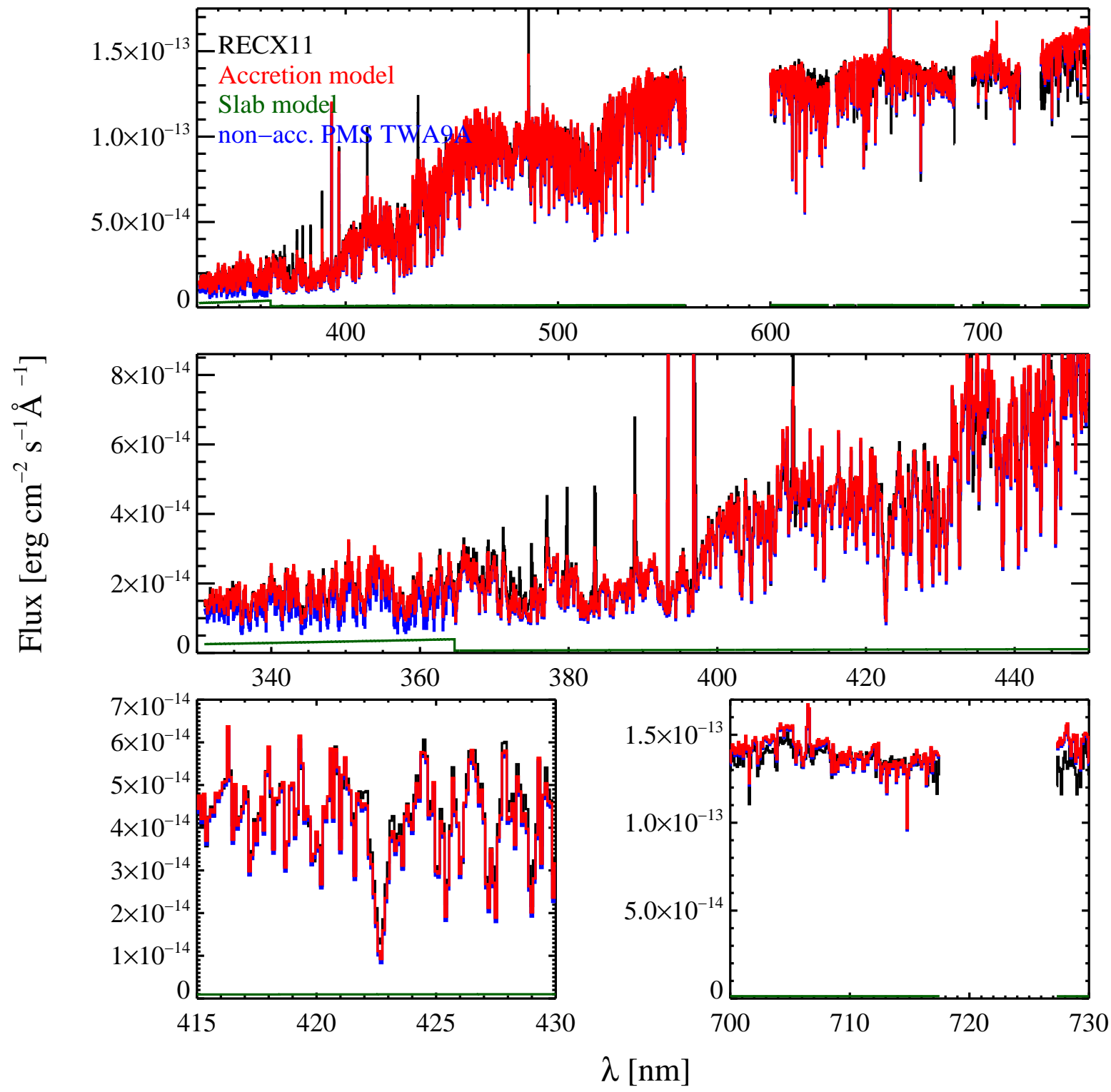

Fig. A.5. Accretion model to PMS RECX 11, using as a basis the non-accreting PMS TWA9A (Manara et al. 2013b). Colors as in Fig. A.1. 


\section{Appendix B: Emission lines}

We report fluxes and equivalent widths of the $\mathrm{H} \alpha$ and $\mathrm{H} \beta$ transitions; the He I $\lambda 402.6$, HeI $\lambda 447.1$, He I $\lambda 501.5, \mathrm{He} \mathrm{I} \lambda 587.5$, He I $\lambda 667.8$, He I $\lambda 706.5$ transitions; and the HeIFeI $\lambda 492.2$ emission feature (Alcalá et al. 2014). We include the detections of the [O I] $\lambda 557.7,[\mathrm{O} \mathrm{I}] \lambda 630.0$, and [O I] $\lambda 636.3$ forbidden transitions.

Table B.1. Fluxes and equivalent widths of forbidden oxygen transitions.

\begin{tabular}{lrrrrrr}
\hline \hline Object & $\begin{array}{r}f_{[\mathrm{OI}] \lambda 557.7} \\
{\left[\mathrm{erg} \mathrm{s}^{-1} \mathrm{~cm}^{-2}\right]}\end{array}$ & $\begin{array}{r}E W_{[\mathrm{OI}]} \lambda 557.7 \\
{[\AA]}\end{array}$ & $\begin{array}{r}f_{[\mathrm{OI}] \lambda 630.0} \\
{\left[\mathrm{erg} \mathrm{s}^{-1} \mathrm{~cm}^{-2}\right]}\end{array}$ & $\begin{array}{r}E W_{[\mathrm{OI}]} \lambda 630.0 \\
{[\AA]}\end{array}$ & $\begin{array}{r}f_{[\mathrm{OI}] \lambda 636.3} \\
{\left[\mathrm{erg} \mathrm{s}^{-1} \mathrm{~cm}^{-2}\right]}\end{array}$ & $\begin{array}{r}E W_{\text {[OI] }} \lambda 636.3 \\
{[\AA]}\end{array}$ \\
\hline J0836 & $<9.56 \mathrm{e}-18$ & - & $<2.92 \mathrm{e}-17$ & - & $<5.84 \mathrm{e}-17$ & - \\
RECX1 & $<1.24 \mathrm{e}-14$ & - & $<9.35 \mathrm{e}-15$ & - & $<6.00 \mathrm{e}-15$ & - \\
J0838 & $<3.63 \mathrm{e}-17$ & - & $<1.26 \mathrm{e}-16$ & - & $<1.72 \mathrm{e}-16$ & - \\
J0841 & $<1.30 \mathrm{e}-17$ & - & $6.65( \pm 0.93) \mathrm{e}-16$ & $-1.18 \pm 0.18$ & $<6.42 \mathrm{e}-17$ & - \\
RECX3 & $<1.62 \mathrm{e}-16$ & - & $<3.61 \mathrm{e}-16$ & - & $<5.48 \mathrm{e}-16$ & - \\
RECX4 & $<9.36 \mathrm{e}-16$ & - & $<1.14 \mathrm{e}-15$ & - & $<1.08 \mathrm{e}-15$ & - \\
RECX5 & $9.34( \pm 3.45) \mathrm{e}-16$ & $-0.27 \pm 0.10$ & $<3.77 \mathrm{e}-16$ & - & $<4.85 \mathrm{e}-16$ & - \\
RECX6 & $<3.03 \mathrm{e}-16$ & - & $<3.26 \mathrm{e}-16$ & - & $<4.46 \mathrm{e}-16$ & - \\
RECX7 & $<5.86 \mathrm{e}-15$ & - & $<5.63 \mathrm{e}-15$ & - & $<9.11 \mathrm{e}-15$ & - \\
J0843 & $6.94( \pm 0.47) \mathrm{e}-15$ & $-1.27 \pm 0.08$ & $7.18( \pm 0.32) \mathrm{e}-14$ & $-12.91 \pm 0.48$ & $2.40( \pm 0.20) \mathrm{e}-14$ & $-3.62 \pm 0.35$ \\
J0844 & $1.35( \pm 0.51) \mathrm{e}-16$ & $-0.73 \pm 0.30$ & $<4.04 \mathrm{e}-17$ & - & $<6.13 \mathrm{e}-17$ & - \\
RECX9 & $1.64( \pm 0.29) \mathrm{e}-15$ & $-0.47 \pm 0.09$ & $<2.98 \mathrm{e}-16$ & - & $<3.95 \mathrm{e}-16$ & - \\
RECX10 & $<1.66 \mathrm{e}-15$ & - & $<1.86 \mathrm{e}-15$ & - & $<1.59 \mathrm{e}-15$ & - \\
RECX11 & $<5.16 \mathrm{e}-15$ & - & $<4.62 \mathrm{e}-15$ & - & $<2.37 \mathrm{e}-15$ & - \\
\hline
\end{tabular}

Table B.2. Fluxes and equivalent widths of helium transitions (1).

\begin{tabular}{lrrrrrr}
\hline \hline Object & $\begin{array}{r}f_{\text {Hel } \lambda 402.6} \\
{\left[\mathrm{erg} \mathrm{s}^{-1} \mathrm{~cm}^{-2}\right]}\end{array}$ & $\begin{array}{r}E W_{\text {HeI } \lambda 402.6} \\
{[\AA]}\end{array}$ & $\begin{array}{r}f_{\text {Hel } \lambda 447.1} \\
{\left[\mathrm{erg} \mathrm{s}^{-1} \mathrm{~cm}^{-2}\right]}\end{array}$ & $\begin{array}{r}E W_{\text {HeI } \lambda 447.1} \\
{[\AA]}\end{array}$ & $\begin{array}{r}f_{\text {HeI } \lambda 501.5} \\
{\left[\mathrm{erg} \mathrm{s}^{-1} \mathrm{~cm}^{-2}\right]}\end{array}$ & $\begin{array}{r}E W_{\text {Hel }} \lambda 501.5 \\
{[\AA]}\end{array}$ \\
\hline J0836 & $<1.54 \mathrm{e}-17$ & - & $1.08( \pm 0.23) \mathrm{e}-16$ & $-0.74 \pm 0.17$ & $<1.07 \mathrm{e}-17$ & - \\
RECX1 & $<1.38 \mathrm{e}-14$ & - & $<1.27 \mathrm{e}-14$ & - & $<1.40 \mathrm{e}-14$ & - \\
J0838 & $<4.74 \mathrm{e}-17$ & - & $<3.29 \mathrm{e}-17$ & - & $<2.40 \mathrm{e}-17$ & - \\
J0841 & $9.42( \pm 4.74) \mathrm{e}-17$ & $-0.61 \pm 0.32$ & $<1.32 \mathrm{e}-17$ & - & $<1.65 \mathrm{e}-17$ & - \\
RECX3 & $<1.87 \mathrm{e}-16$ & - & $<1.46 \mathrm{e}-16$ & - & $<2.25 \mathrm{e}-16$ & - \\
RECX4 & $<5.80 \mathrm{e}-16$ & - & $<7.96 \mathrm{e}-16$ & - & $<1.03 \mathrm{e}-15$ & - \\
RECX5 & $1.73( \pm 0.34) \mathrm{e}-15$ & $-2.10 \pm 0.49$ & $2.22( \pm 0.32) \mathrm{e}-15$ & $-1.33 \pm 0.22$ & $<9.46 \mathrm{e}-17$ & - \\
RECX6 & $<2.30 \mathrm{e}-16$ & - & $<1.71 \mathrm{e}-16$ & - & $<2.91 \mathrm{e}-16$ & - \\
RECX7 & $<7.74 \mathrm{e}-15$ & - & $<8.81 \mathrm{e}-15$ & - & $<6.56 \mathrm{e}-15$ & - \\
J0843 & $6.01( \pm 0.49) \mathrm{e}-15$ & $-2.34 \pm 0.19$ & $1.13( \pm 0.06) \mathrm{e}-14$ & $-3.09 \pm 0.14$ & $2.34( \pm 0.11) \mathrm{e}-14$ & $-5.69 \pm 0.22$ \\
J0844 & $6.66( \pm 0.64) \mathrm{e}-16$ & $-5.08 \pm 0.63$ & $1.28( \pm 0.07) \mathrm{e}-15$ & $-8.08 \pm 0.71$ & $3.30( \pm 0.31) \mathrm{e}-16$ & $-2.27 \pm 0.24$ \\
RECX9 & $9.03( \pm 1.99) \mathrm{e}-16$ & $-0.88 \pm 0.20$ & $1.33( \pm 0.17) \mathrm{e}-15$ & $-0.81 \pm 0.10$ & $<9.75 \mathrm{e}-17$ & - \\
RECX10 & $<8.34 \mathrm{e}-16$ & - & $<1.26 \mathrm{e}-15$ & - & $<1.62 \mathrm{e}-15$ & - \\
RECX11 & $<5.13 \mathrm{e}-15$ & - & $<5.13 \mathrm{e}-15$ & - & $<6.05 \mathrm{e}-15$ & - \\
RECX12 & $2.68( \pm 0.63) \mathrm{e}-15$ & $-0.52 \pm 0.13$ & $4.69( \pm 1.23) \mathrm{e}-15$ & $-0.42 \pm 0.11$ & $<5.55 \mathrm{e}-16$ & - \\
\hline
\end{tabular}


Table B.3. Fluxes and equivalent widths of helium transitions (2).

\begin{tabular}{lrrrrrr}
\hline \hline Object & $\begin{array}{r}f_{\text {HeI } \lambda 587.5} \\
{\left[\mathrm{erg} \mathrm{s}^{-1} \mathrm{~cm}^{-2}\right]}\end{array}$ & $\begin{array}{r}E W_{\text {HeI } \lambda 587.5} \\
{[\AA]}\end{array}$ & $\begin{array}{r}f_{\text {HeI } \lambda 667.8} \\
{\left[\mathrm{erg} \mathrm{s}^{-1} \mathrm{~cm}^{-2}\right]}\end{array}$ & $\begin{array}{r}E W_{\text {HeI } \lambda 667.8} \\
{[\AA]}\end{array}$ & $\begin{array}{r}f_{\text {Hel } \lambda 706.5} \\
{\left[\mathrm{erg} \mathrm{s}^{-1} \mathrm{~cm}^{-2}\right]}\end{array}$ & $\begin{array}{r}E W_{\text {HeI } \lambda 706.5} \\
{[\AA]}\end{array}$ \\
\hline J0836 & $<7.02 \mathrm{e}-17$ & - & $<6.24 \mathrm{e}-17$ & - & $<9.36 \mathrm{e}-17$ & - \\
RECX1 & $<1.08 \mathrm{e}-14$ & - & $<3.21 \mathrm{e}-15$ & - & $<4.20 \mathrm{e}-15$ & - \\
J0838 & $<2.15 \mathrm{e}-16$ & - & $<1.64 \mathrm{e}-16$ & - & $<1.78 \mathrm{e}-16$ & - \\
J0841 & $<9.16 \mathrm{e}-17$ & - & $<8.71 \mathrm{e}-17$ & - & $<9.63 \mathrm{e}-17$ & - \\
RECX3 & $<5.20 \mathrm{e}-16$ & - & $<4.37 \mathrm{e}-16$ & - & $<9.34 \mathrm{e}-16$ & - \\
RECX4 & $<1.52 \mathrm{e}-15$ & - & $<8.19 \mathrm{e}-16$ & - & $<1.87 \mathrm{e}-15$ & - \\
RECX5 & $4.64( \pm 1.29) \mathrm{e}-15$ & $-1.86 \pm 0.51$ & $3.97( \pm 1.33) \mathrm{e}-15$ & $-0.95 \pm 0.38$ & $<4.67 \mathrm{e}-16$ & - \\
RECX6 & $<5.48 \mathrm{e}-16$ & - & $<3.28 \mathrm{e}-16$ & - & $<6.29 \mathrm{e}-16$ & - \\
RECX7 & $<6.53 \mathrm{e}-15$ & - & $<1.71 \mathrm{e}-15$ & - & $<3.19 \mathrm{e}-15$ & - \\
J0843 & $2.25( \pm 0.19) \mathrm{e}-14$ & $-4.70 \pm 0.35$ & $6.37( \pm 1.24) \mathrm{e}-15$ & $-0.85 \pm 0.19$ & $<4.91 \mathrm{e}-16$ & - \\
J0844 & $1.45( \pm 0.22) \mathrm{e}-15$ & $-9.95 \pm 1.44$ & $1.00( \pm 0.13) \mathrm{e}-15$ & $-4.66 \pm 1.13$ & $6.17( \pm 1.04) \mathrm{e}-16$ & $-1.56 \pm 0.28$ \\
RECX9 & $4.76( \pm 1.03) \mathrm{e}-15$ & $-1.66 \pm 0.35$ & $<2.51 \mathrm{e}-16$ & - & $<4.02 \mathrm{e}-16$ & - \\
RECX10 & $<1.97 \mathrm{e}-15$ & - & $<9.02 \mathrm{e}-16$ & - & $<1.84 \mathrm{e}-15$ & - \\
RECX11 & $<5.20 \mathrm{e}-15$ & - & $<1.53 \mathrm{e}-15$ & - & $<2.24 \mathrm{e}-15$ & - \\
RECX12 & $1.43( \pm 0.19) \mathrm{e}-14$ & $-0.70 \pm 0.09$ & $<7.17 \mathrm{e}-16$ & - & $<1.52 \mathrm{e}-15$ & - \\
\hline
\end{tabular}

Table B.4. Fluxes and equivalent widths of helium (3), $\mathrm{H} \alpha$, and $\mathrm{H} \beta$ transitions.

\begin{tabular}{lrrrrrr}
\hline \hline Object & $\begin{array}{r}f_{\mathrm{HeIFel} \lambda 492.2} \\
{\left[\mathrm{erg} \mathrm{s}^{-1} \mathrm{~cm}^{-2}\right]}\end{array}$ & $\begin{array}{r}E W_{\mathrm{HeIFel}} \lambda 492.2 \\
{[\AA]}\end{array}$ & $\begin{array}{r}f_{\mathrm{H} \alpha} \lambda 656.3 \\
{\left[\mathrm{erg} \mathrm{s}^{-1} \mathrm{~cm}^{-2}\right]}\end{array}$ & $\begin{array}{r}E W_{\mathrm{H} \alpha \lambda 656.3} \\
{[\AA]}\end{array}$ & $\begin{array}{r}f_{\mathrm{H} \beta \lambda 486.1} \\
{\left[\mathrm{erg} \mathrm{s}^{-1} \mathrm{~cm}^{-2}\right]}\end{array}$ & $\begin{array}{r}E W_{\mathrm{H} \beta} \lambda 486.1 \\
{[\AA]}\end{array}$ \\
\hline J0836 & $<1.36 \mathrm{e}-17$ & - & $1.25( \pm 0.06) \mathrm{e}-14$ & $-13.38 \pm 0.72$ & $2.10( \pm 0.09) \mathrm{e}-15$ & $-11.13 \pm 0.37$ \\
RECX1 & $<1.17 \mathrm{e}-14$ & - & $3.19( \pm 0.27) \mathrm{e}-13$ & $-1.05 \pm 0.09$ & $<1.22 \mathrm{e}-14$ & - \\
J0838 & $<2.66 \mathrm{e}-17$ & - & $2.23( \pm 0.12) \mathrm{e}-14$ & $-11.98 \pm 0.77$ & $4.19( \pm 0.20) \mathrm{e}-15$ & $-10.42 \pm 0.40$ \\
J0841 & $<1.85 \mathrm{e}-17$ & - & $1.10( \pm 0.06) \mathrm{e}-14$ & $-9.45 \pm 0.62$ & $2.32( \pm 0.11) \mathrm{e}-15$ & $-8.26 \pm 0.29$ \\
RECX3 & $<2.13 \mathrm{e}-16$ & - & $3.94( \pm 0.38) \mathrm{e}-14$ & $-2.73 \pm 0.28$ & $7.97( \pm 0.61) \mathrm{e}-15$ & $-1.91 \pm 0.14$ \\
RECX4 & $<9.90 \mathrm{e}-16$ & - & $1.85( \pm 0.11) \mathrm{e}-13$ & $-4.24 \pm 0.26$ & $4.50( \pm 0.30) \mathrm{e}-14$ & $-2.68 \pm 0.16$ \\
RECX5 & $<8.64 \mathrm{e}-17$ & - & $8.68( \pm 0.42) \mathrm{e}-14$ & $-13.50 \pm 0.68$ & $2.92( \pm 0.12) \mathrm{e}-14$ & $-14.70 \pm 0.35$ \\
RECX6 & $<2.80 \mathrm{e}-16$ & - & $8.93( \pm 0.47) \mathrm{e}-14$ & $-5.32 \pm 0.24$ & $2.28( \pm 0.11) \mathrm{e}-14$ & $-4.03 \pm 0.14$ \\
RECX7 & $<7.28 \mathrm{e}-15$ & - & $1.65( \pm 0.21) \mathrm{e}-13$ & $-0.79 \pm 0.10$ & $<7.20 \mathrm{e}-15$ & - \\
J0843 & $2.40( \pm 0.11) \mathrm{e}-14$ & $-4.99 \pm 0.11$ & $9.90( \pm 0.40) \mathrm{e}-13$ & $-94.45 \pm 2.63$ & $1.75( \pm 0.07) \mathrm{e}-13$ & $-42.61 \pm 0.68$ \\
J0844 & $6.64( \pm 0.39) \mathrm{e}-16$ & $-3.26 \pm 0.16$ & $4.40( \pm 0.18) \mathrm{e}-14$ & $-102.20 \pm 5.45$ & $1.40( \pm 0.06) \mathrm{e}-14$ & $-82.07 \pm 1.98$ \\
RECX9 & $<9.69 \mathrm{e}-17$ & - & $9.60( \pm 0.43) \mathrm{e}-14$ & $-12.60 \pm 0.47$ & $2.10( \pm 0.09) \mathrm{e}-14$ & $-10.73 \pm 0.30$ \\
RECX10 & $<1.31 \mathrm{e}-15$ & - & $9.14( \pm 1.05) \mathrm{e}-14$ & $-1.68 \pm 0.20$ & $2.76( \pm 0.38) \mathrm{e}-14$ & $-1.15 \pm 0.16$ \\
RECX11 & $<5.27 \mathrm{e}-15$ & - & $1.16( \pm 0.05) \mathrm{e}-12$ & $-8.32 \pm 0.29$ & $2.56( \pm 0.21) \mathrm{e}-13$ & $-2.73 \pm 0.22$ \\
RECX12 & $<6.36 \mathrm{e}-16$ & - & $3.05( \pm 0.13) \mathrm{e}-13$ & $-8.41 \pm 0.22$ & $1.06( \pm 0.04) \mathrm{e}-13$ & $-8.15 \pm 0.16$ \\
\hline
\end{tabular}

